\title{
A COMPARATIVE STUDY OF HOSPITAL SUSTAINABILITY RATING SYSTEMS
}

\author{
Mahmoud Fathy Ahmed*, Moaaz Ahmed Abd-Allah and Salama Mostafa El-Nagar \\ Architectural Department, Shoubra Faculty of Engineering, Benha University, Shoubra, \\ Cairo, Egypt \\ *Corresponding Author E-mail: Mahmoud.Fathy@Feng.Bu.Edu.Eg
}

\begin{abstract}
Sustainability is one of the most important goals that global society tries to achieve, many of environmental organizations think about how to evaluate sustainability in different types of building, so sustainability rating systems are published, some of these rating systems are general, and some of them are specializing in a certain building types, Hospitals are one of these types, many rating systems with different items and credits are used to evaluate sustainability in hospitals. So, it will be useful to compare between these rating systems to define similarities and differences between them, then to specify which the best one for hospitals. The conclusion of this paper is, despite the apparent similarity of hospital rating systems in items and credits, Breeam is the most comprehensive one, as it contains the largest number of sustainability assessment criteria as well as Breeam which includes all sustainability elements (environmental - social - economic).
\end{abstract}

\section{KEY WORDS: Sustainability, Green Buildings, Hospitals, Rating Systems, LEED,} Breeam, and Green Star

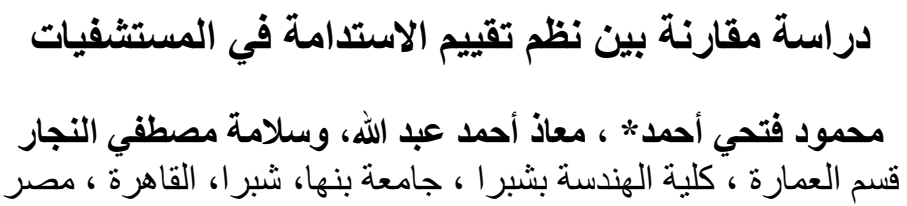

E-mail : Mahmoud.Fathy@Feng.Bu.Edu.Eg البريد الاليكتروني للباحث الرئيسي:

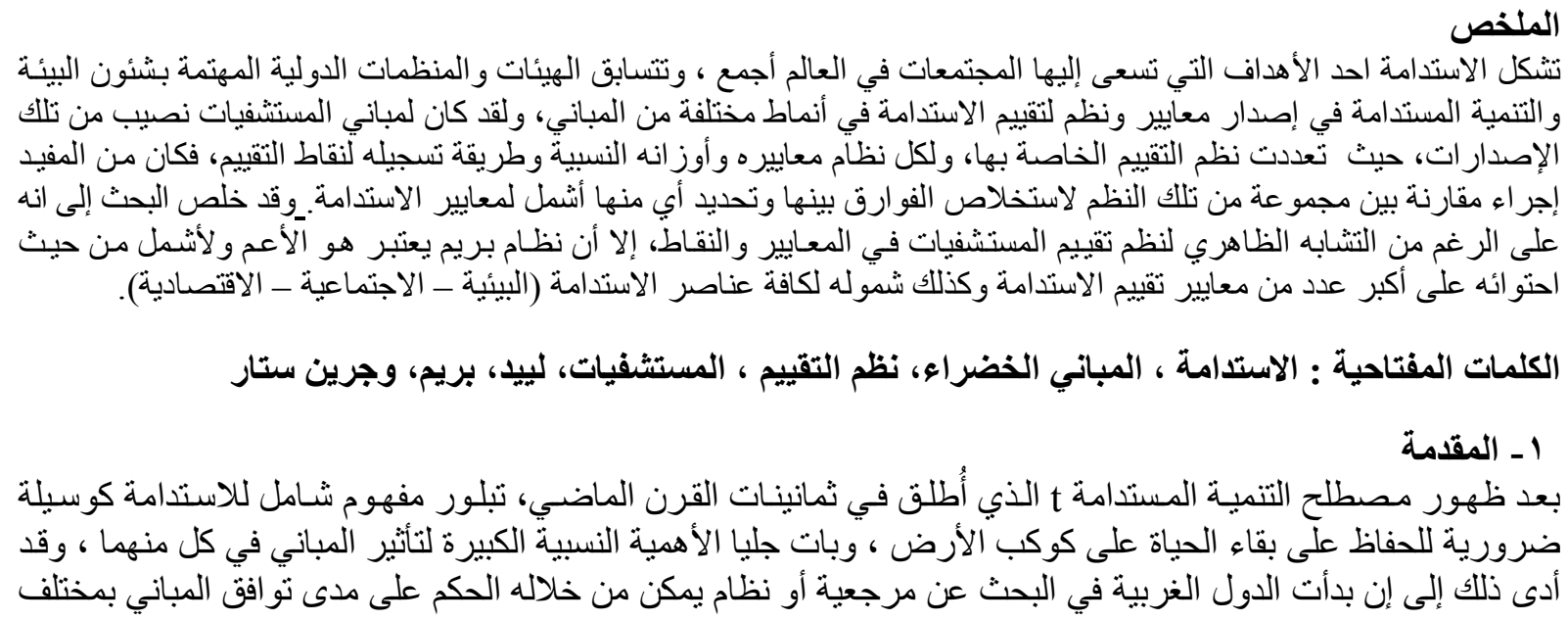


نو عياتها مع أهداف الحفاظ على البيئة والاقتصاد في استهلاك الطاقة والموارد، لذا فقد بدأت نللك الدول في وضع نظم لتقيهيم

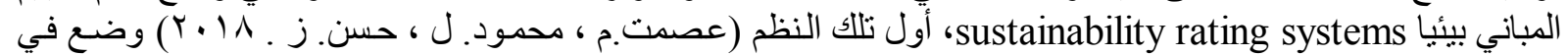

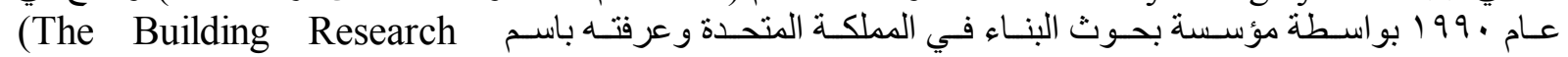
Establishment Environmental Assessment Method - BREEAM) ، الو لايات المتحدة الأمريكية بعمل نظام (Leadership in Energy and Environmental Design - LEED)

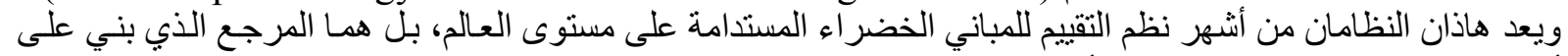

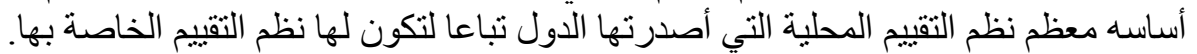

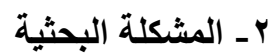

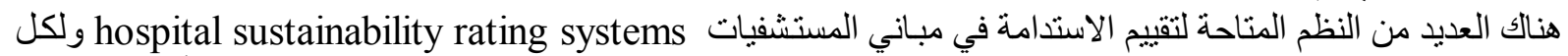

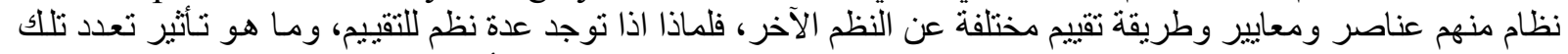

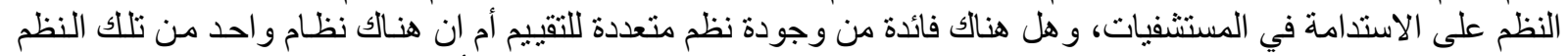

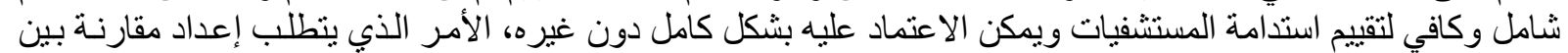
تلاك النظ لتحديد فائدة تعددها من عدمه التهام

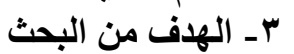

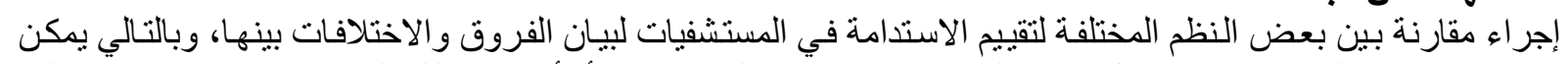

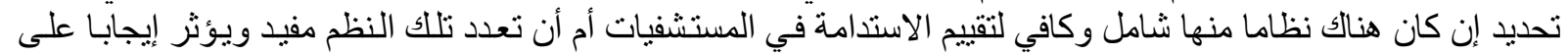

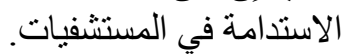

\section{ـ ـ الاستذامة في مباني المستثفيات المبات}

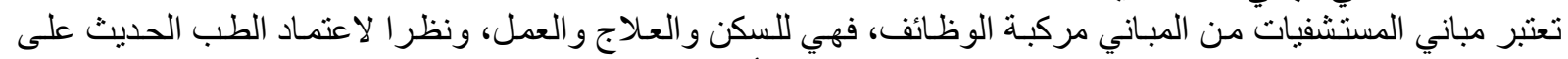

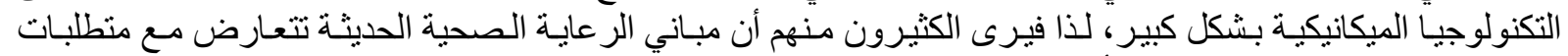

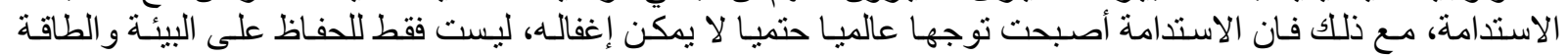

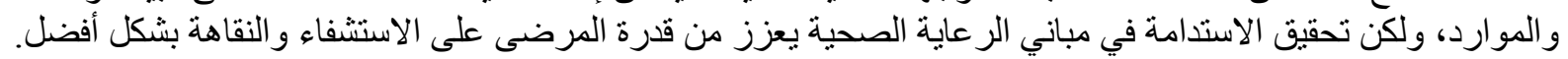

(Bensalem 2011)

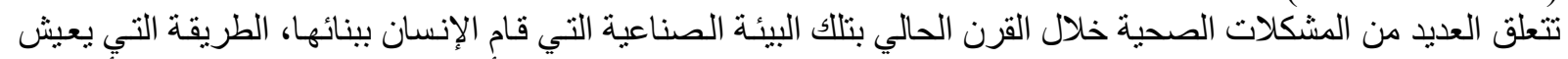

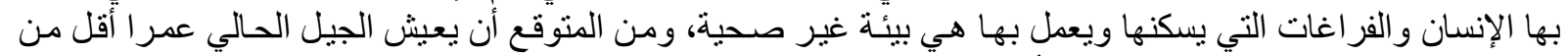

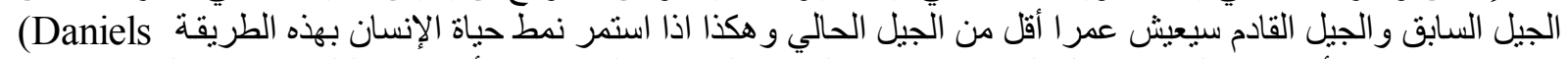

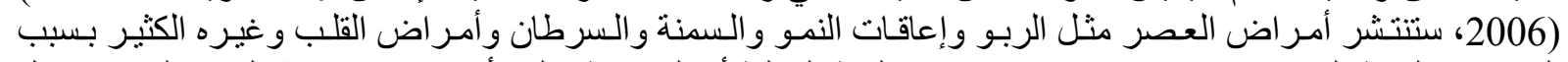

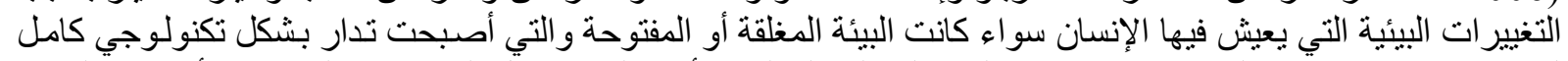

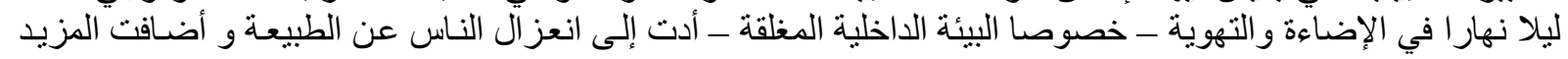
من الضغوطٌ النفسية و العصبية عليهم.

احتياج الإنسان لمباني المستشفيات لن يكن للعلاج فحسب، فما فائدة العلاج إذا وبعد انتهاؤه يعود الإنسان لنفس البيئة التي لئي

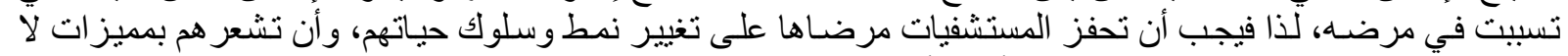

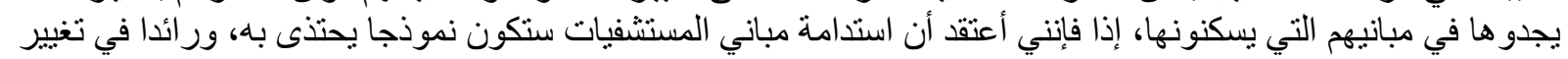

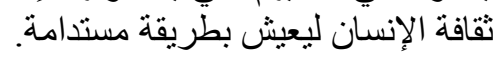
هـ متطلبات تحقيق الاستدامة في مباني المستثفيات الإنة

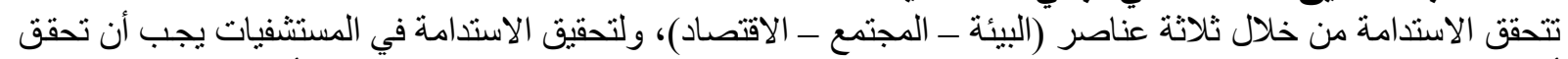

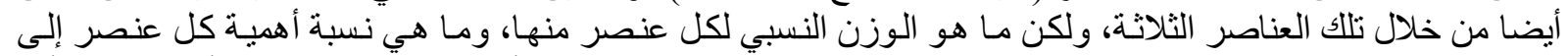

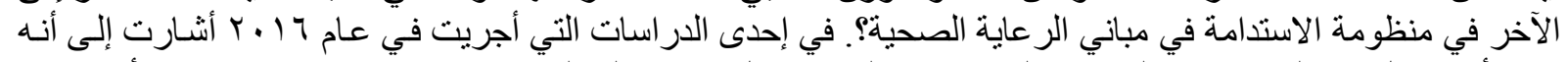

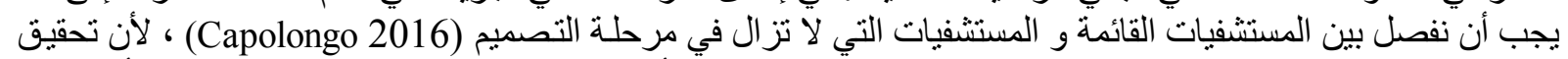

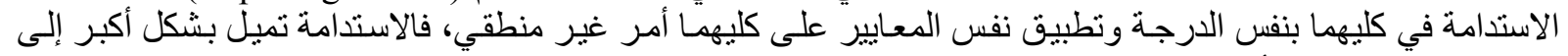

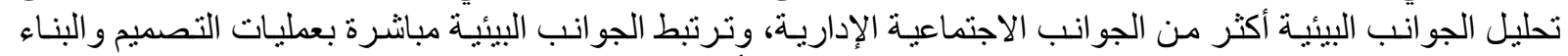

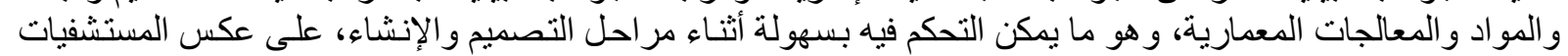

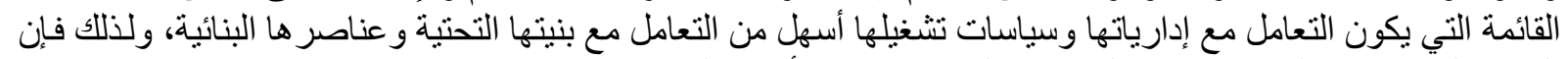

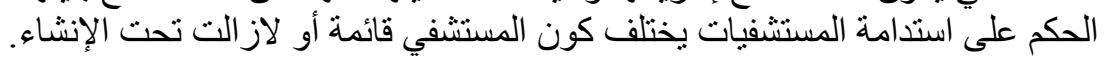



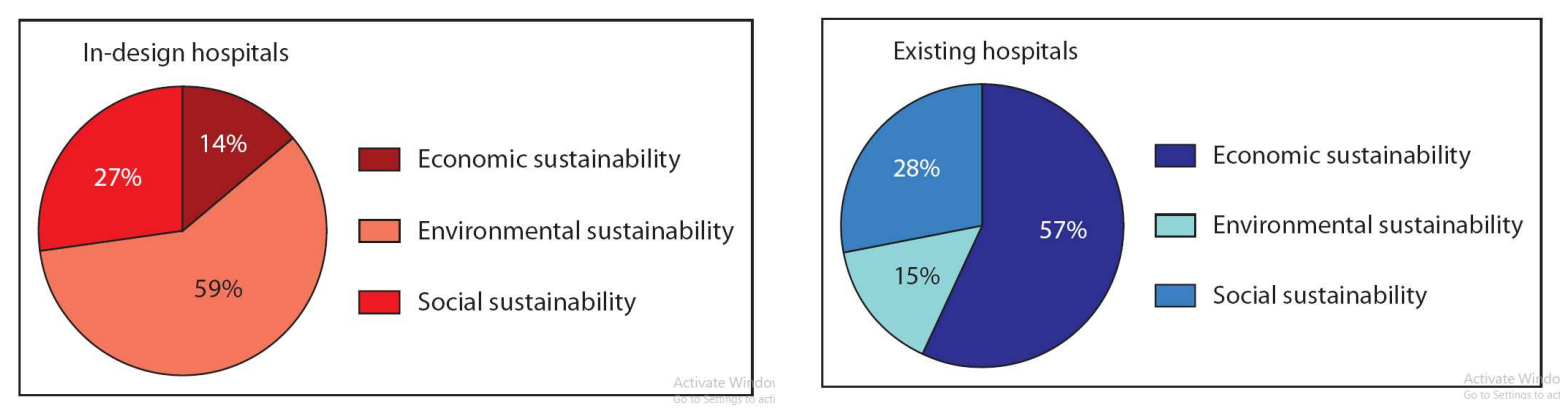

الشكل ا نسب عناصر الاستدامة في المستثفيات القائمة و الجديدة المصدر (Capolongo 2016)

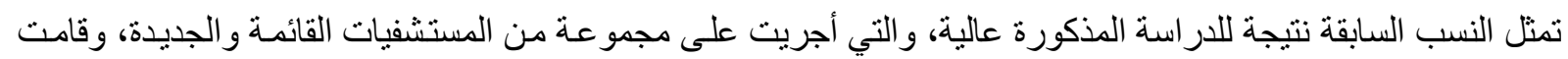

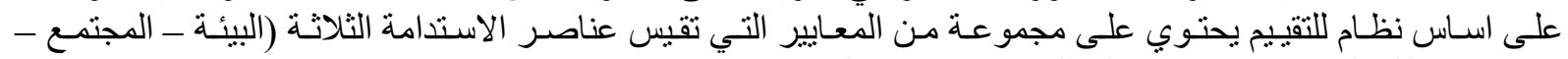

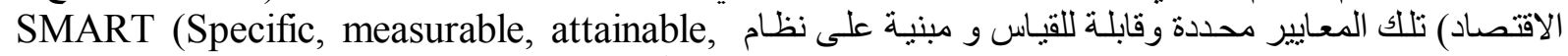
relevant and timely)

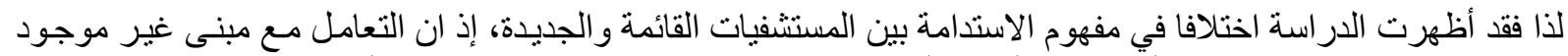

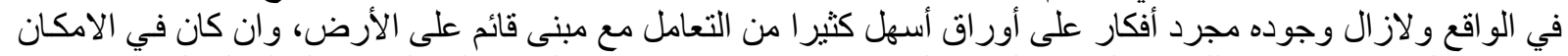

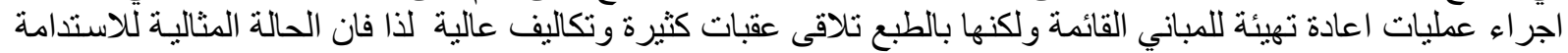

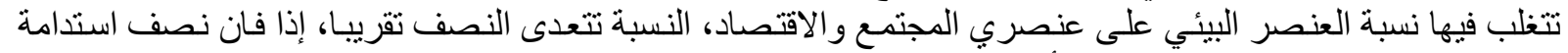

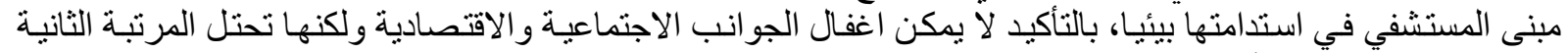

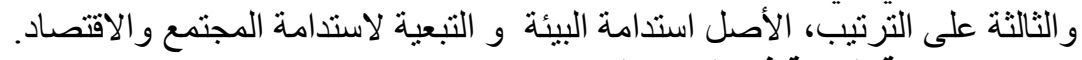

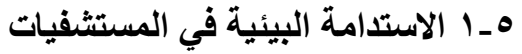

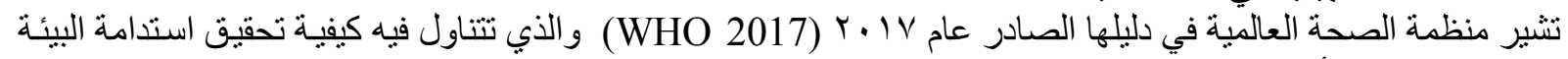

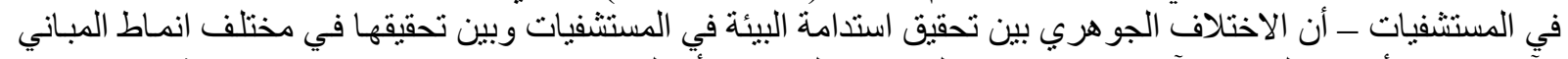

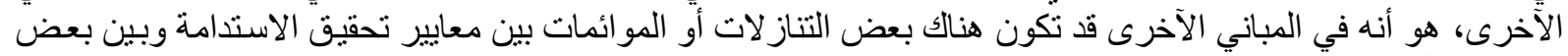

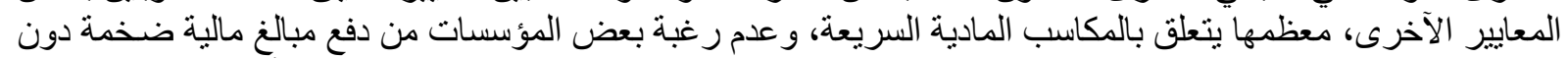

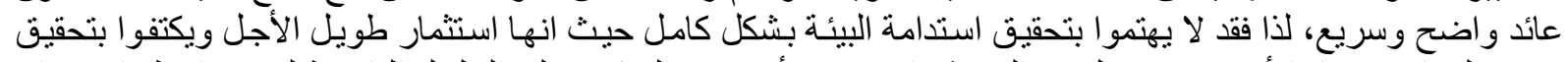

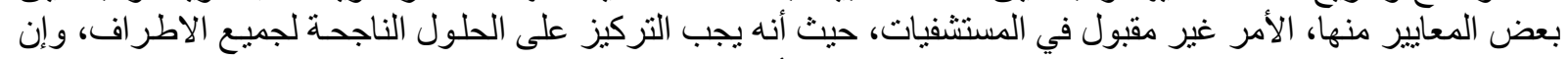

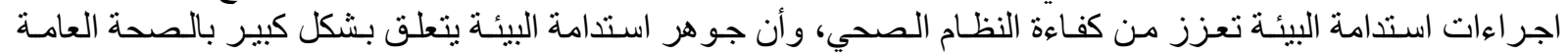

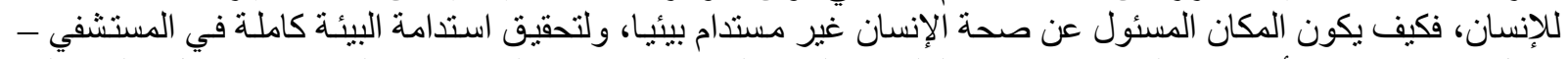

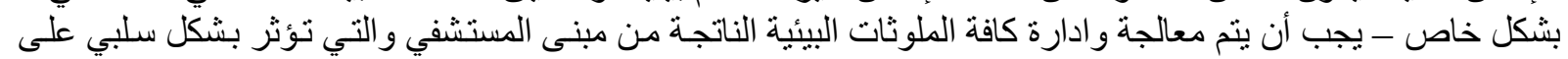

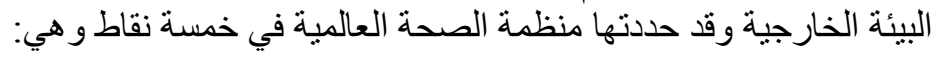
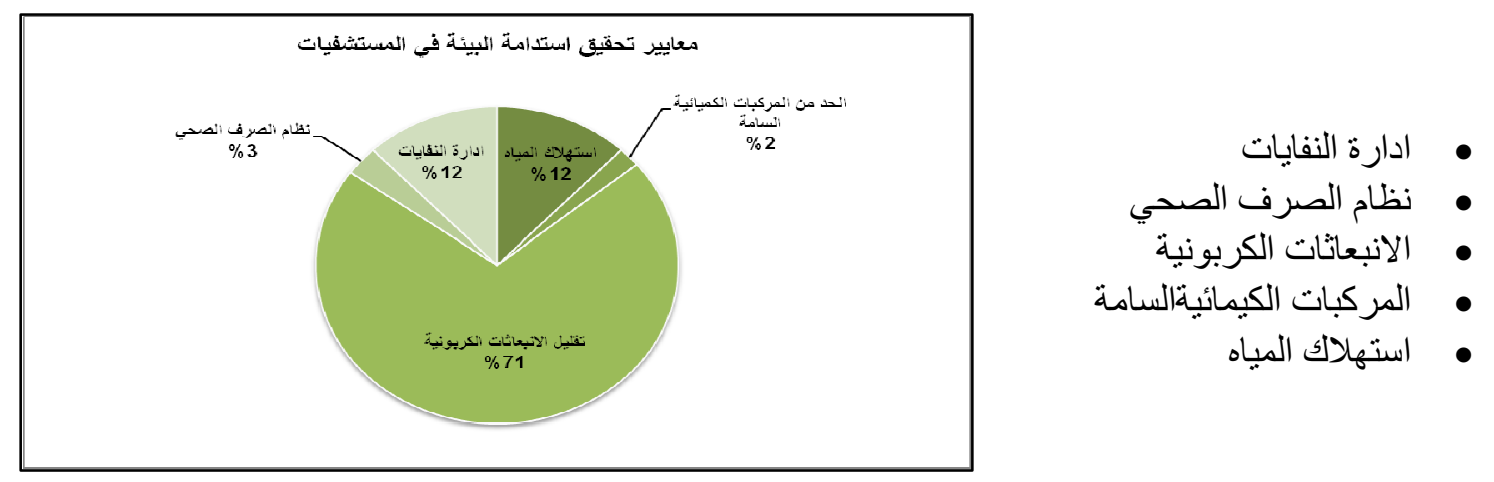

الشكل r نسب عناصر استدامة البيئة في المستشفيات المصدر (Buffoli 2015) 
تعرف r_o

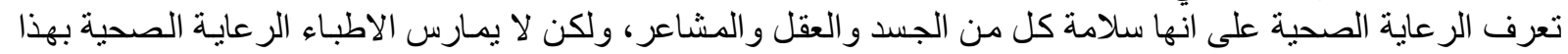

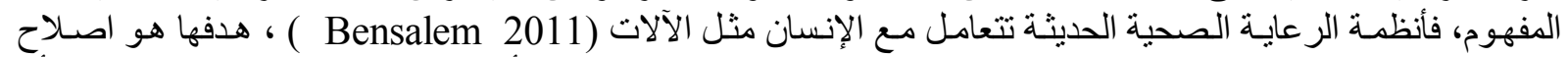

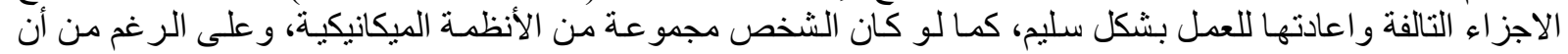

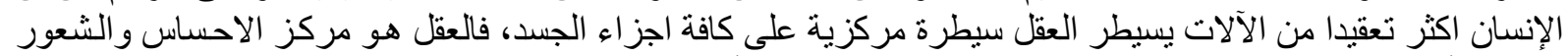

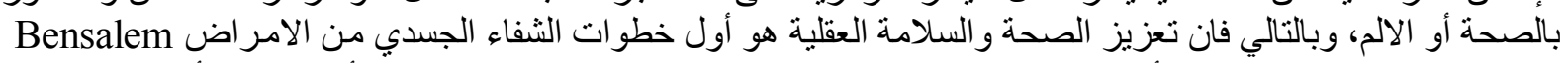

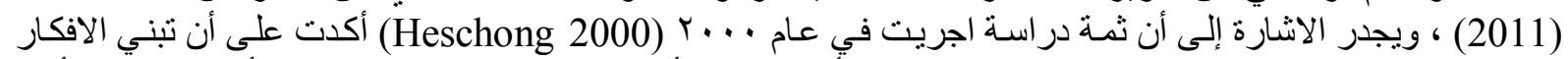

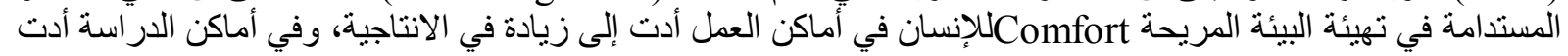
إلى زيادة في معدلات التحصيل فئة العلمي للطلاب.

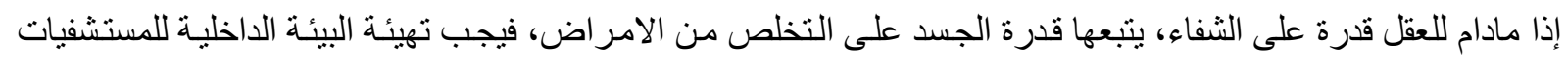

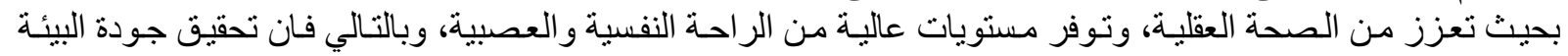

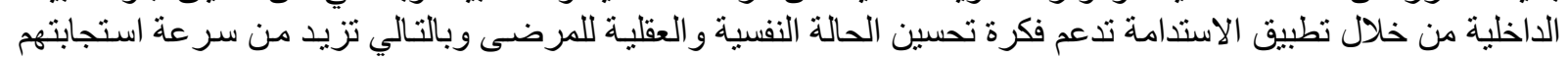

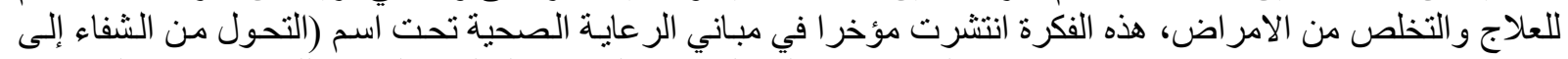

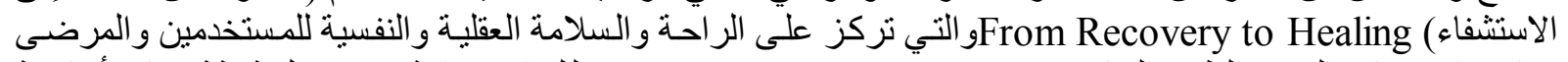

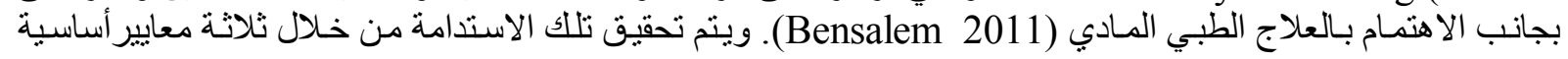

معايير تحفيق استداهة المجتمع في المستثفيات

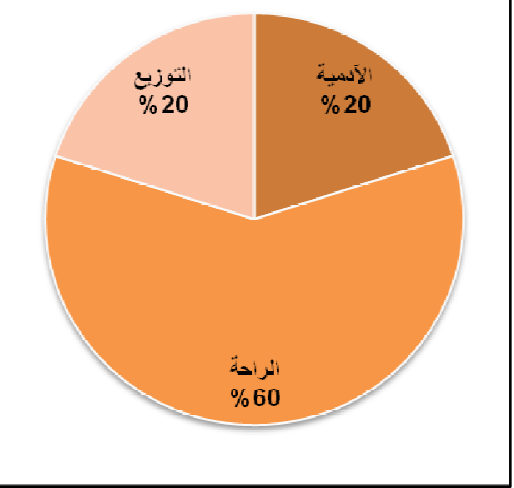

الآدمية Humanization: المعيار الذي يقيم بيئة وسياسات المستشفي.

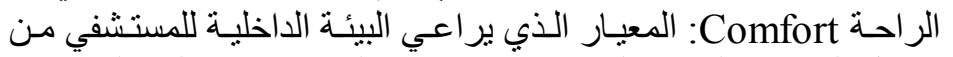

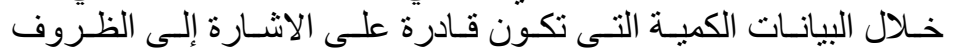
المناخية الداخلية.

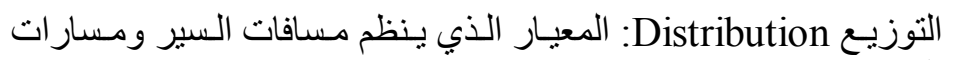
الحركة وتوزيع الاقسام.

الشكل r نسب عناصر استدامة المجتمع في المستثفيات

المصدر (Buffoli 2015)

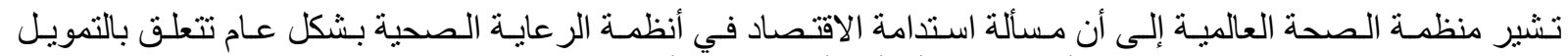

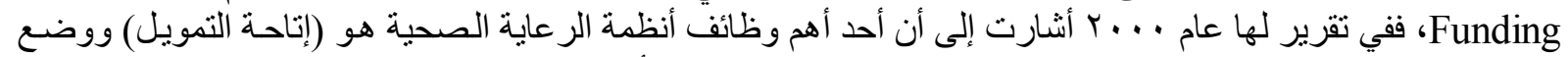

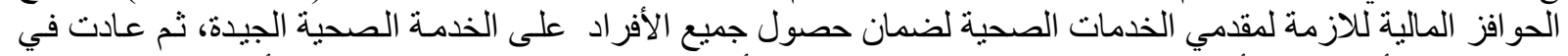

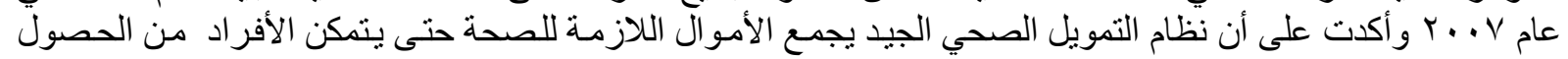

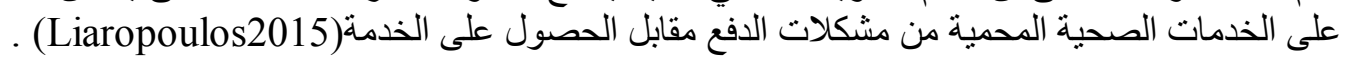

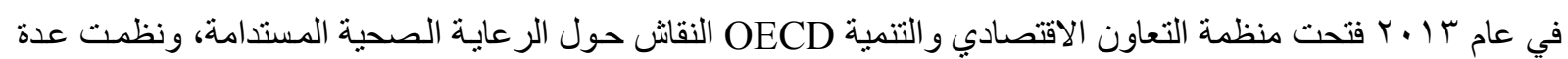

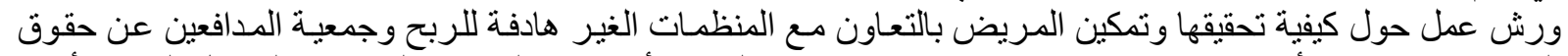

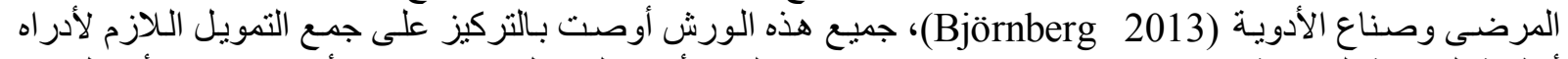

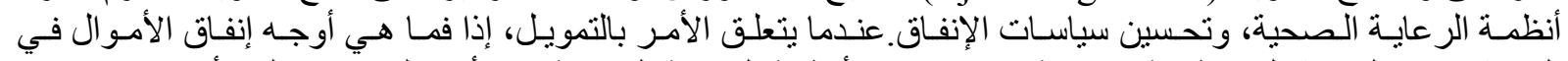

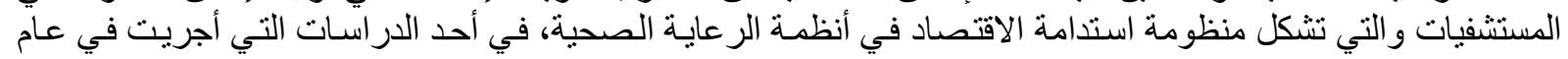

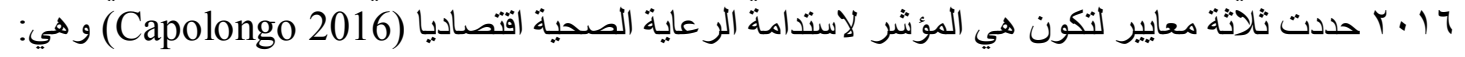




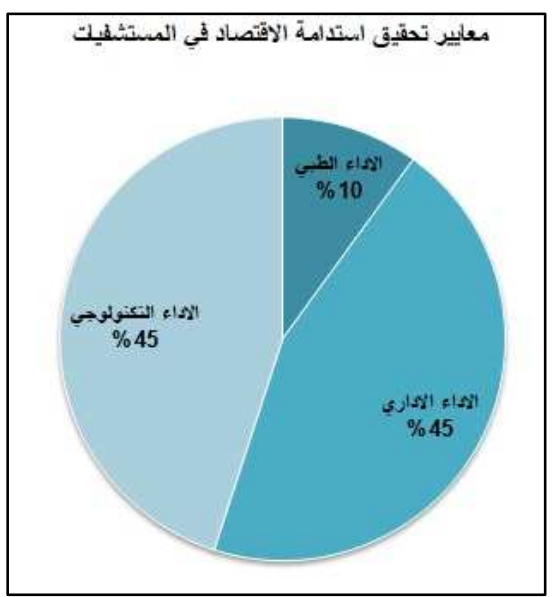

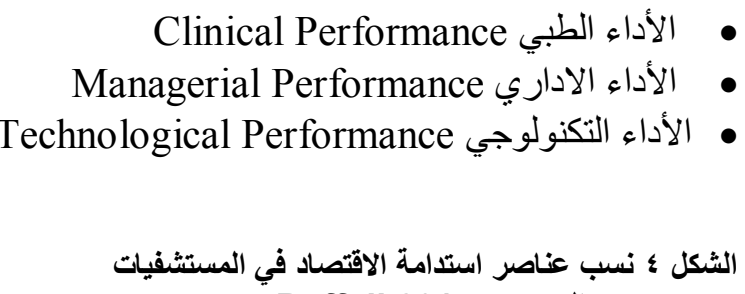

(Buffoli 2015) (المصدر

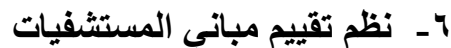

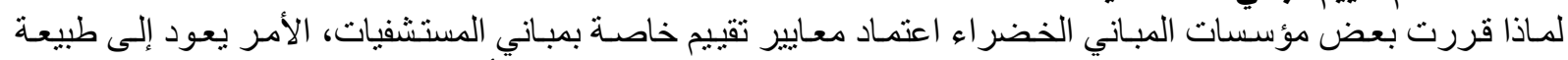

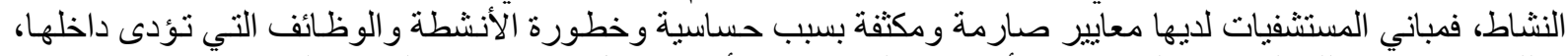

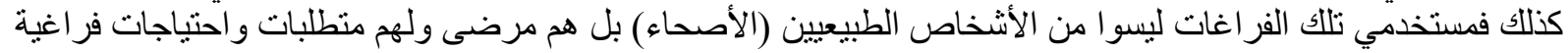

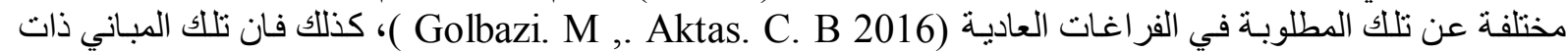

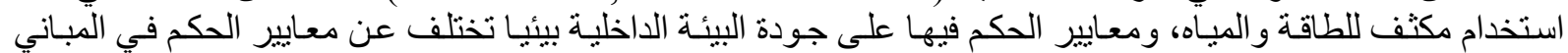

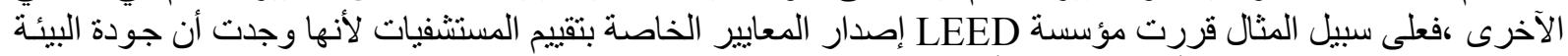

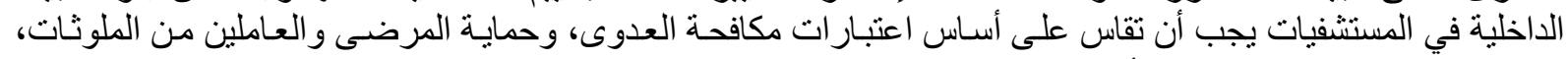
و إجر اءات التهوية الجيدة، لذا فان أنظمة تقييم المباني العادية لا تناسب الطبيعية الوظيفية لمباني المستشفيات.

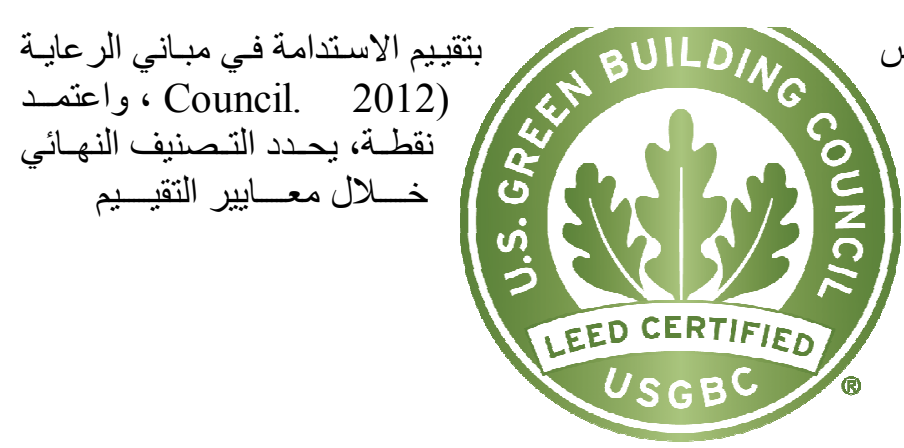

LEED for Healthcare $-v$

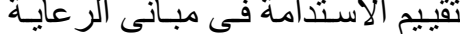
( Council. 2012)

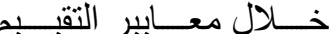

(U.S Green Building Council. 2012)

بلاتيني Platinum

أعلى من • ^ نقطة
صدر الإصـار الأول مـن نظسام LEED الخـاص

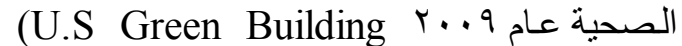

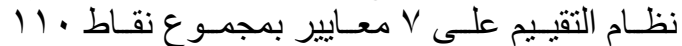

للمبنى طبقا لمجمو ع النقاط التي تحصل علير عليها من

.(Golbazi. M ,. Aktas. C. B 2016)

جدول r معايير تقييم LEED HC) - المصدر (U.S Green Building Council. 2012)

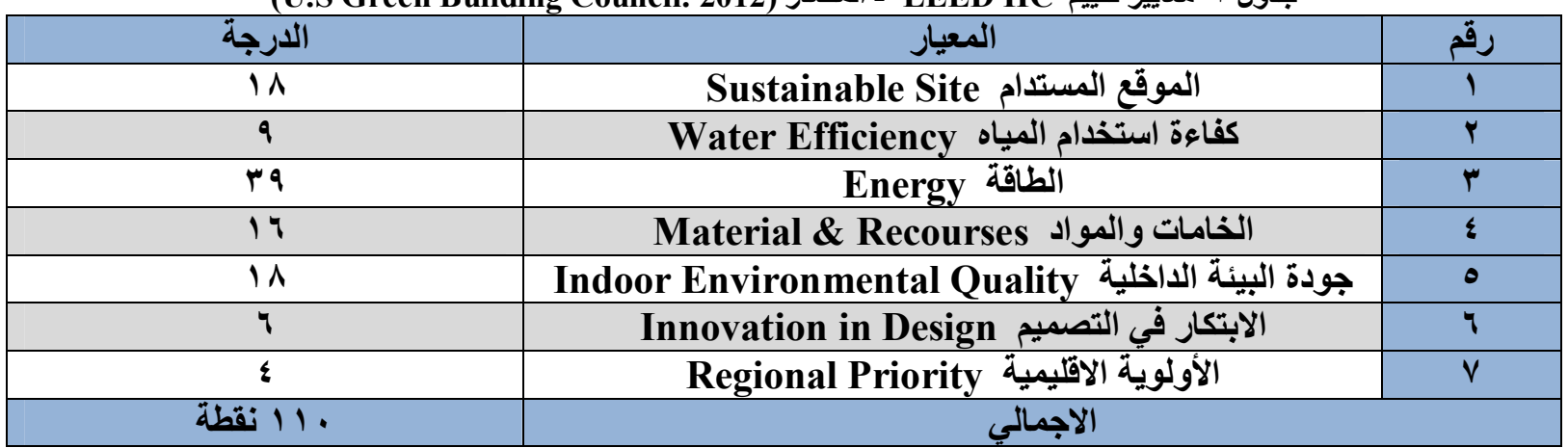

معتمد جertified

من · إلى 9 ؟ نقطة 


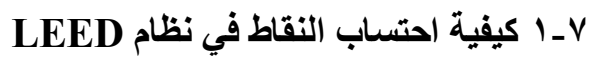

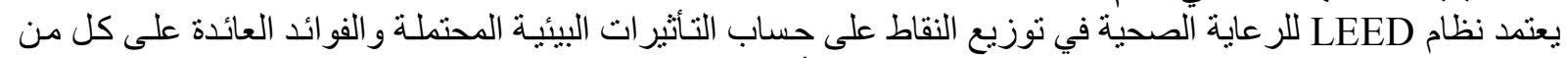

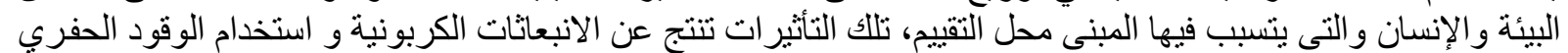

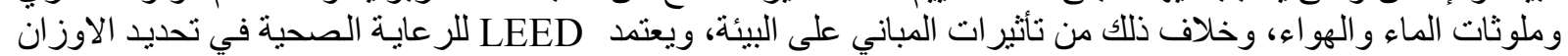

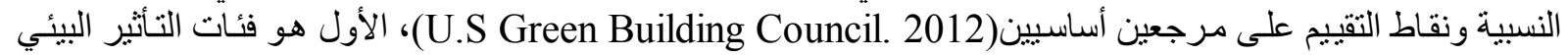
Tool for the Reduction and Assessment of Chemical and Other Environmental ) TRACI -1 National Institute ) NIST Impacts ) ( of Standards and Technology

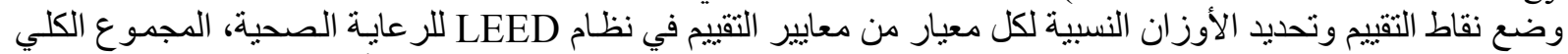

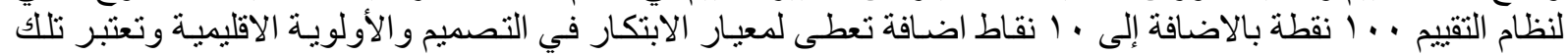

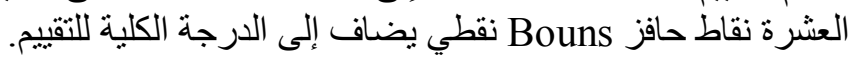

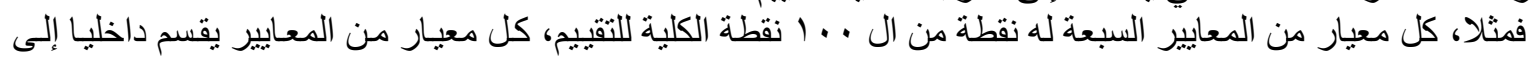

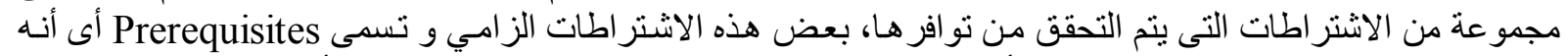

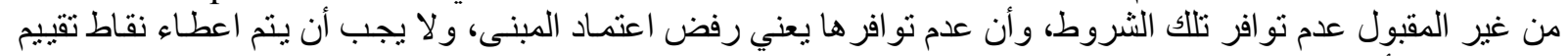

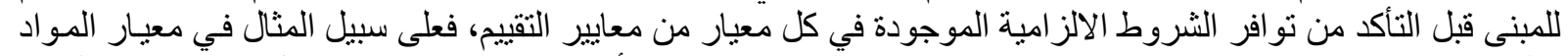

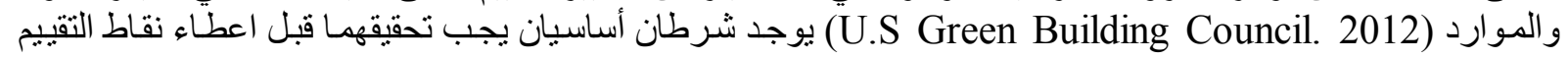

تخزين وجمع المو اد القابلة لاعادة التدوير : أى أنه يجب عمل نظام فصل للنفايات بحيث يمكن الاستفادة من نفايات المو اد القابلة للاعادة الاستخدام.

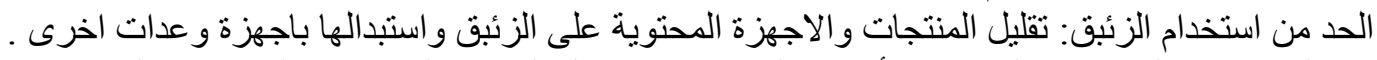

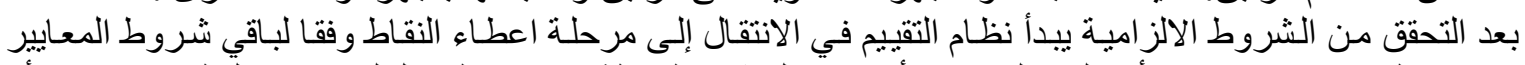

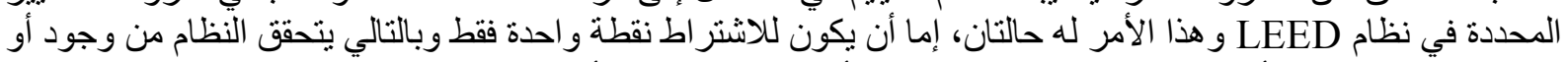

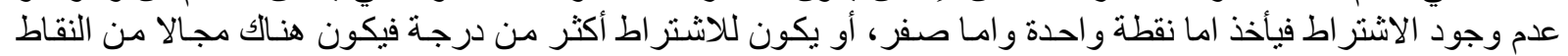
تعطى لكل حالة من حالات تحقق الاشتر اطنت اطنة

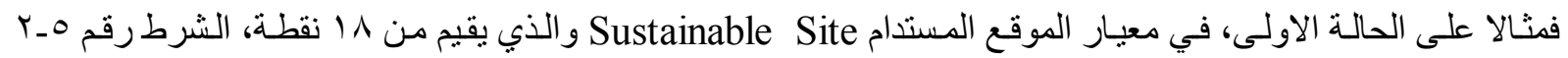

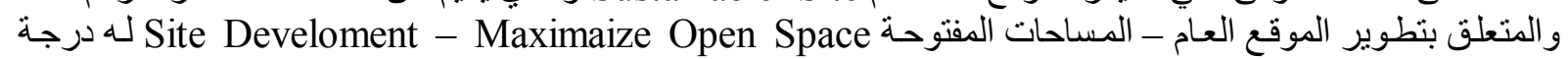

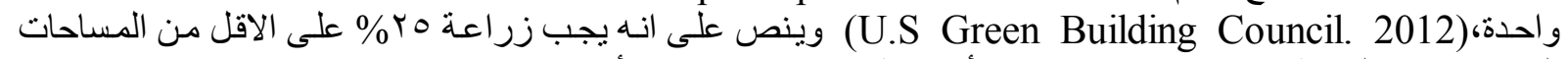
المفتوحة، هذا الشرط غير نسبي، فإما محقق فيأخد نقطة و إما غير محقق فيأخذ صفر.

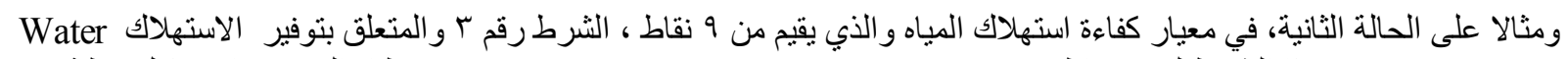
Use Reduction

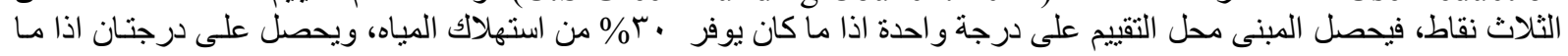

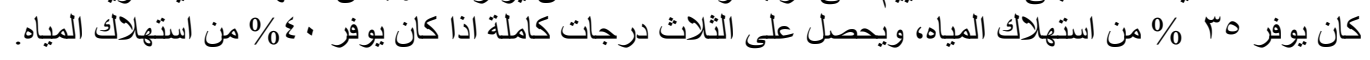

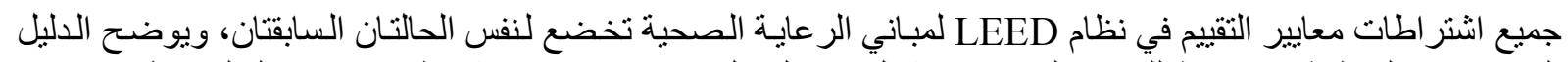

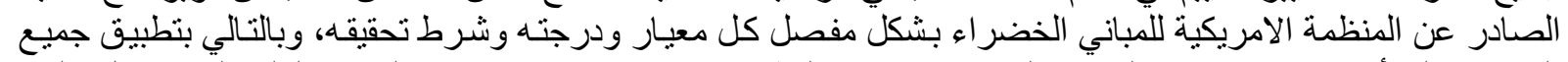

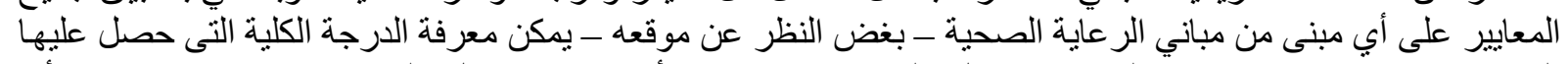

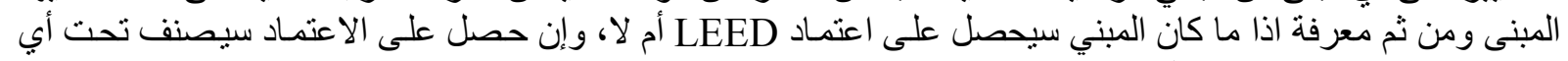
تصنيف (بلاتيني او ذهبي ... الخ).

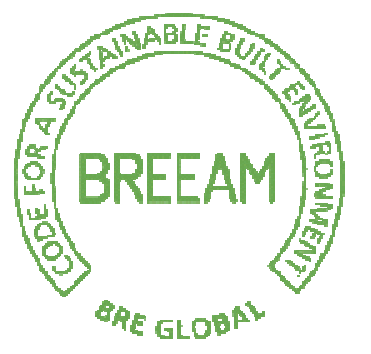

BREEAM for Healthcare $-\wedge$

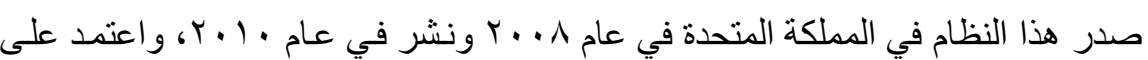

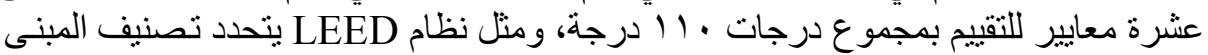

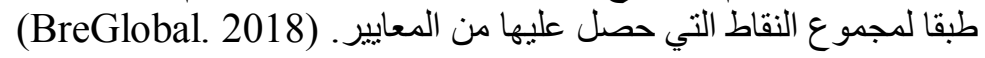


جدول ب تصنيف المباني طبقا لدرجة التقييم لنظام BREEAM HC - المصدر (BreGlobal. 2018)

\begin{tabular}{|c|c|c|c|c|}
\hline $\begin{array}{c}\text { مرموق } \\
\text { Outstanding }\end{array}$ & Excellent ممتاز & $\begin{array}{c}\text { Qيد جدا Gery } \\
\text { Good }\end{array}$ & جيد Good & مقبول Pass \\
\hline هـ نقطة على الاقل & . نقطة على الاقل & هـ نقطة على الاقل & 0 ا نقطة على الاقل & • آنقة على الاقّ \\
\hline
\end{tabular}

جدول ؛ معايير تقييم BREEAM HC المصدر BreGlobal. 2018)

\begin{tabular}{|c|c|c|}
\hline الارجة & المعيار & رقم \\
\hline Ir & الادارة Management & 1 \\
\hline 10 & Health \& Wellbeing الصحة والرفاهية & r \\
\hline 19 & Energy الطاقة & $r$ \\
\hline$\Lambda$ & النقل Transport & $\varepsilon$ \\
\hline 7 & المياه Water & 0 \\
\hline IY.0 & المواد Materials & 7 \\
\hline$V_{.0}$ & النفايات Waste & $\mathrm{V}$ \\
\hline 1. & Land Use استعمال الارض & $\Lambda$ \\
\hline 1. & التلوث Pollution & 9 \\
\hline 1. & Innovation الابتكار & 1. \\
\hline . 11 نقطة & الاجمالي & \\
\hline
\end{tabular}

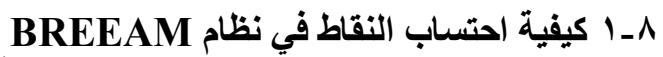

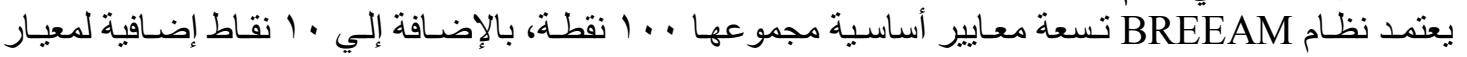

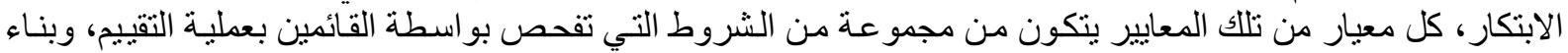

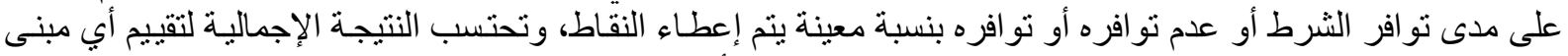

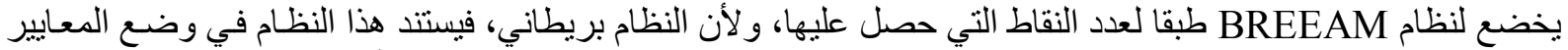

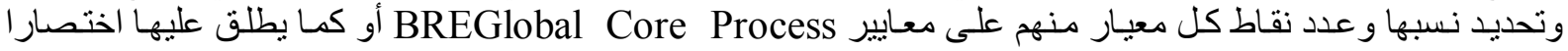
BED5301 و الصادرة عن مؤسسة BREGLOBAL البريطانية، و التي وضعت المعايير القياسية البيئية للبناء المستدام.

.(BreGlobal. 2018)

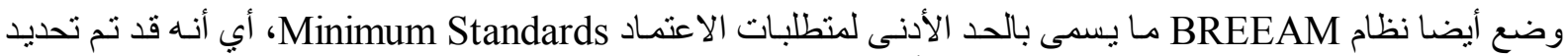

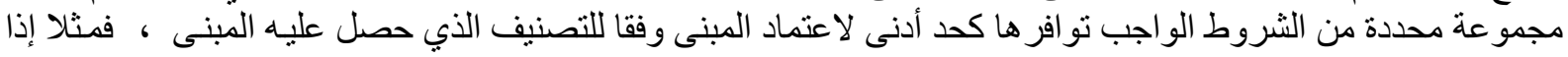

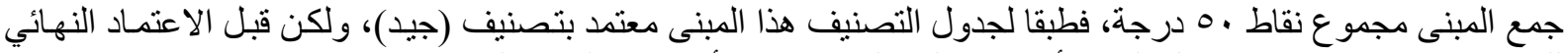

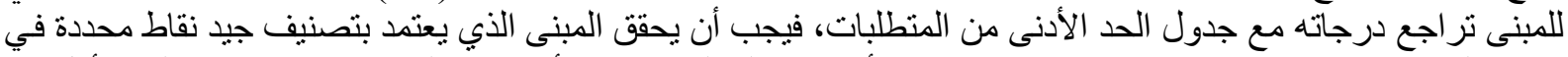

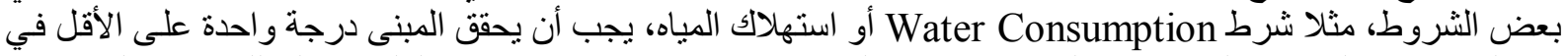

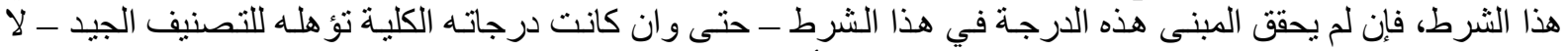
يعتمد المبنى وفق التصنيف (جيد) ويعتمد طبقا للتصنيف الأقل (مقبول) (BreGlobal. 2018). 
جدول • جدول الدد الادني من متطلبات الاعتماد لكل تصنيف لنظام BREEAM- المصدر (BreGlobal. 2018).

\begin{tabular}{|c|c|c|c|c|c|}
\hline & \multicolumn{5}{|c|}{ Minimum standards by BREEAM rating level } \\
\hline BREEAM issue & Pass & Good & Very Good & Excellent & Outstanding \\
\hline $\begin{array}{l}\text { Wat } 01 \text { Water } \\
\text { consumption }\end{array}$ & None & One credit & One credit & One credit & Two credits \\
\hline $\begin{array}{l}\text { Wat } 02 \text { Water } \\
\text { monitoring }\end{array}$ & None & $\begin{array}{l}\text { Criterion } \\
1 \text { only }\end{array}$ & Criterion 1 only & Criterion 1 only & Criterion 1 only \\
\hline $\begin{array}{l}\text { Mat } 03 \\
\text { Responsible } \\
\text { sourcing of } \\
\text { construction } \\
\text { products }\end{array}$ & $\begin{array}{l}\text { Criterion } \\
1 \text { only }\end{array}$ & $\begin{array}{l}\text { Criterion } \\
1 \text { only }\end{array}$ & Criterion 1 only & Criterion 1 only & Criterion 1 only \\
\hline $\begin{array}{l}\text { Wst } 01 \\
\text { Construction } \\
\text { waste } \\
\text { management }\end{array}$ & None & None & None & None & One credit \\
\hline $\begin{array}{l}\text { Wst } 03 \\
\text { Operational } \\
\text { waste }\end{array}$ & None & None & None & One credit & One credit \\
\hline
\end{tabular}

GREEN STAR for Healthcare - 9

نظسام Green star للرعايـة الصحية هو احد نظم التقييم الصادرة عن (مجلس المباني الخضر اء في استرليا) The Green Building Council of Australia (GBCA)

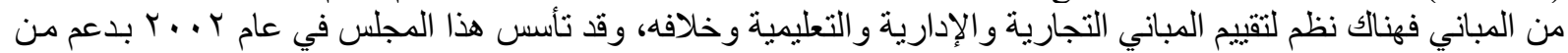

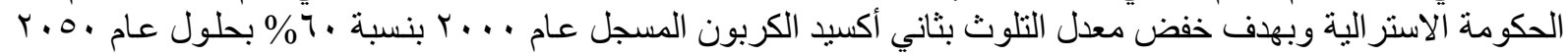

.(Australia Government. 2008)

\section{greenstar Certification}

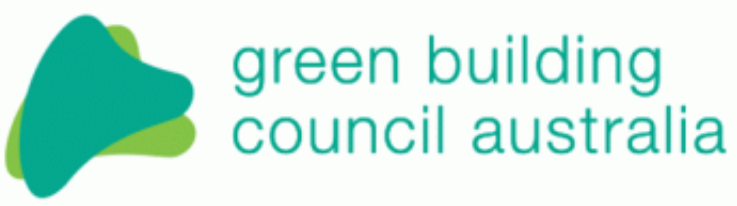

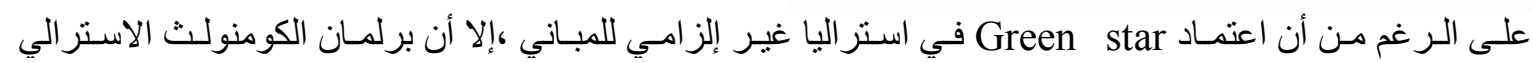

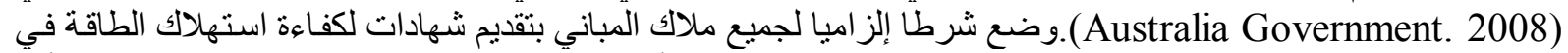

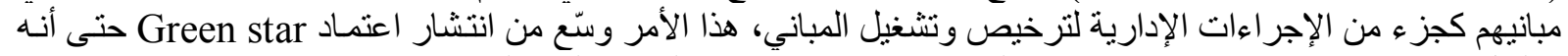

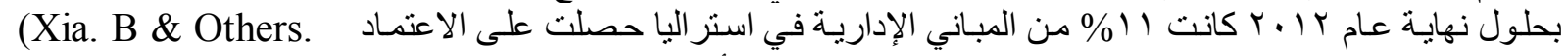

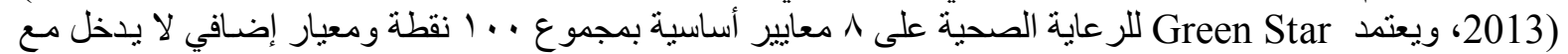

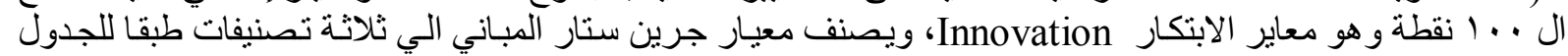

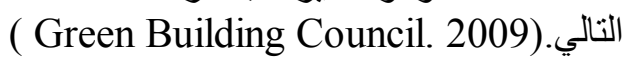

جدول ج تصنيف المباني طبقا لارجة التقييم لنظام Green Star HC المصدر (Green Building Council. 2009)

\begin{tabular}{|c|c|c|}
\hline ا & ه نجوم & ع نجوم \\
\hline اكبر من \& V نقطة & من · \ درجة إلى ع V نقطة & من هـ درجة إلى 9ه نقطة \\
\hline
\end{tabular}


جدول V معايير تقييم Green Star HC) المصدر (Green Building Council. 2009)

\begin{tabular}{|c|c|c|}
\hline الدرجة & المعيار & رقم \\
\hline 9 & Management الادارة & 1 \\
\hline r. & جودة البيئة الداخلية Indoor Environmental Quality & $r$ \\
\hline$r \varepsilon$ & Energy الطاقة & $r$ \\
\hline V & Transport النقل & $\varepsilon$ \\
\hline ir & المياه Water & 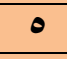 \\
\hline IV & المواد Materials & 7 \\
\hline$\Lambda$ & Land Use استعمال الارض & $\mathrm{V}$ \\
\hline$r$ & Emissions الانبعاثًات الضارة & $\Lambda$ \\
\hline اضافي & Innovation الابتكار & 9 \\
\hline ... & الاجمالى & \\
\hline
\end{tabular}

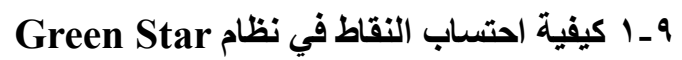

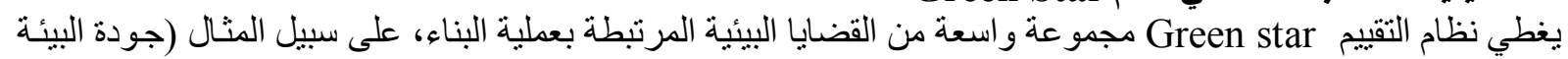

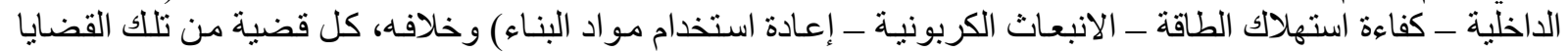

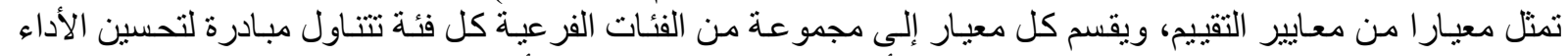

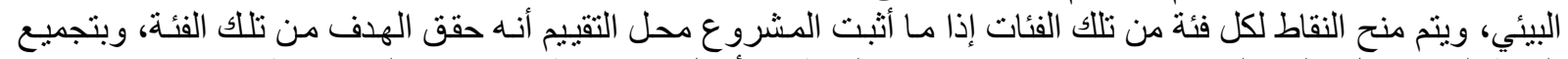

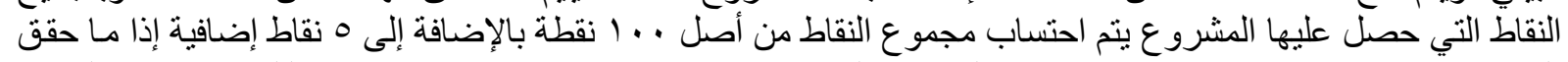

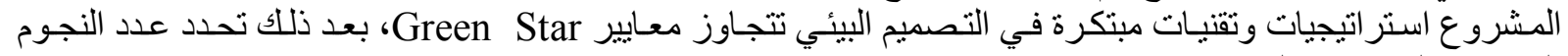

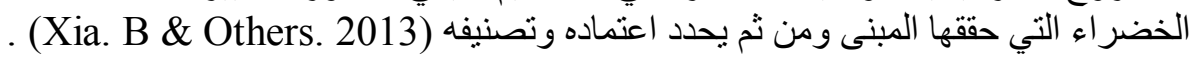

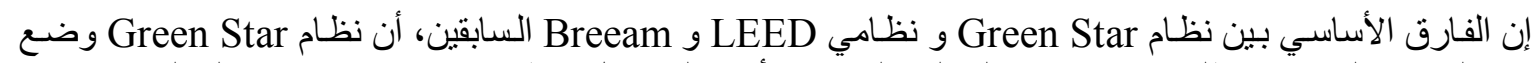

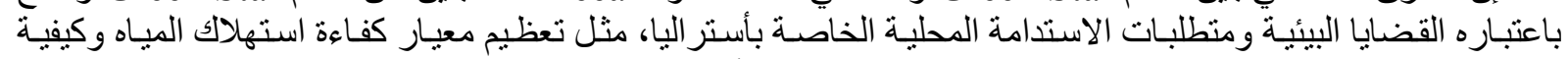

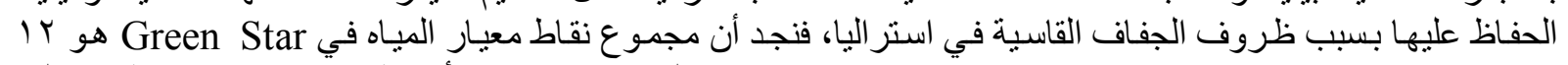

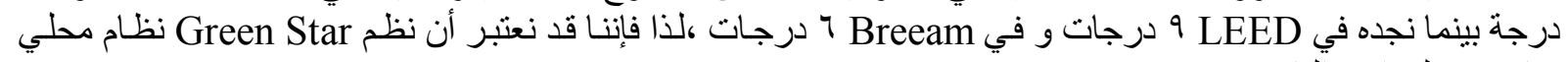

مناسب لإقليم استر أليا. (Green Building Council. 2009) درجة فينات

IGBC for Healthcare- .

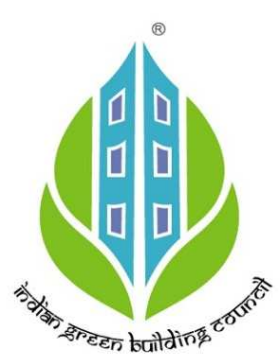

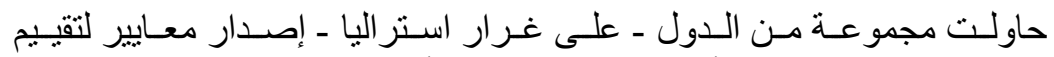

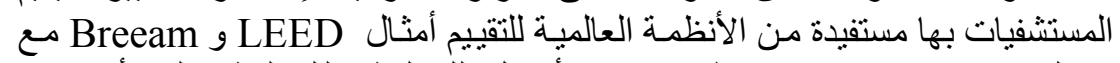

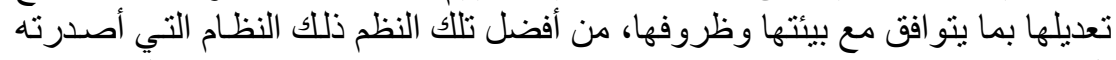

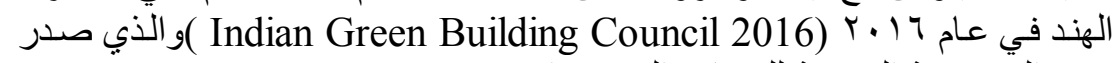

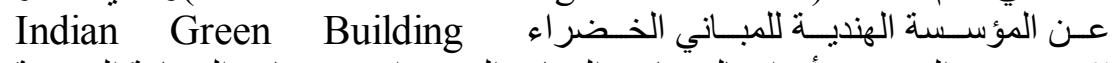
Council

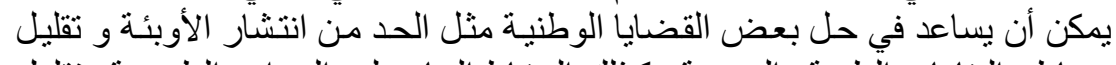

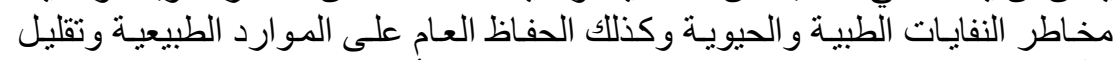

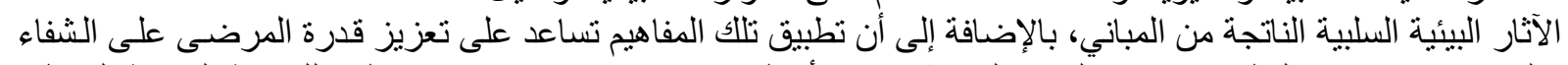

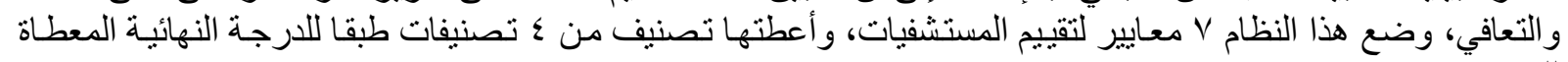
للمستشفي. 
جدول ^ تصنيف المباني طبقا لارجة التقييم لنظام IGBC HC المصدر Indian Green Building Council 2016 )

\begin{tabular}{|c|c|c|c|}
\hline بلاتيني Platinum & ذهبيGold & فضيSilver & معتمد Certified \\
\hline من · ^ إلى · . 1 نقطة & 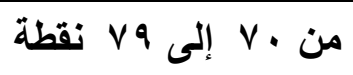 & من · 7 إلى 99 نقطة & من · • إلى 9 ه نقطة \\
\hline
\end{tabular}

جدول ج معايير تقييم IGBC HC المصدر Indian Green Building Council 2016)

\begin{tabular}{|c|c|c|}
\hline الارجة & المعيار & رقم \\
\hline $1 \varepsilon$ & التعقيم والنظافة & 1 \\
\hline rr & جودة البيئة الداخلية & $r$ \\
\hline$r r$ & كفاءة استهلاك الطاقة & $r$ \\
\hline 10 & توفير المياه & $\varepsilon$ \\
\hline 9 & مواد البناء والموارد & 0 \\
\hline 11 & اختيار الموقع & 7 \\
\hline 0 & الابتكار & $\mathrm{V}$ \\
\hline ... & الاجمالي & \\
\hline
\end{tabular}

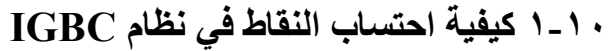

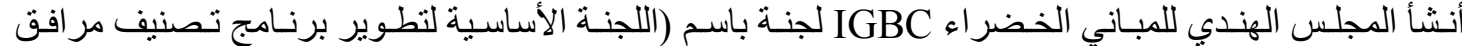

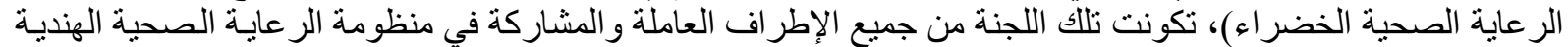

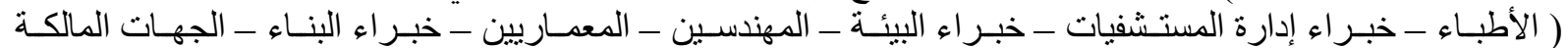

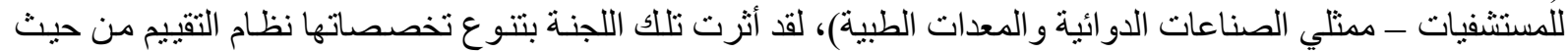

المحتوى وقابلية التطبيق.) Indian Green Building Council 2016)

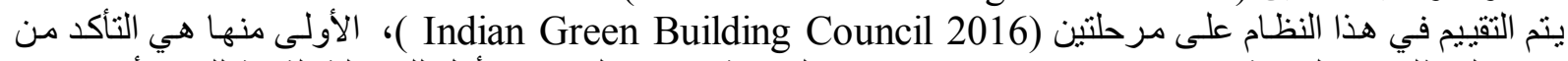

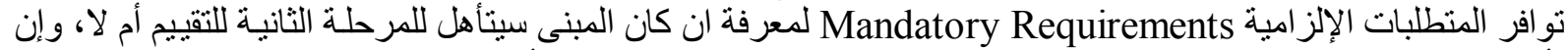

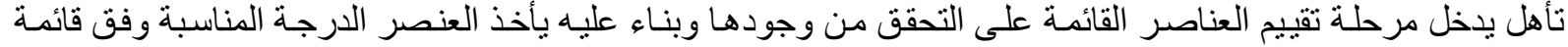

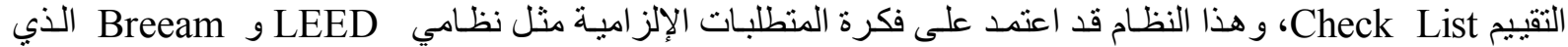
يفرض بعض الشروط الإلز امية التي يجب أن تلتحقى.

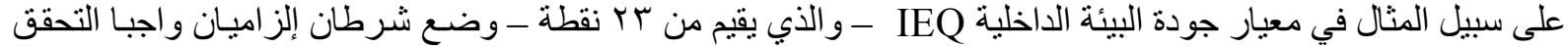

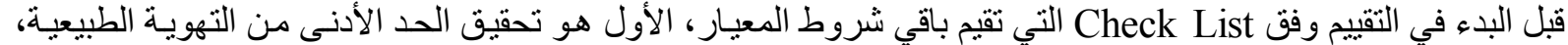

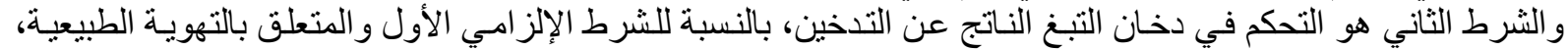
و لأن ليست جميع فر اغات المستشفي قابلة لأن تزود بالتهوية الطبيعية، حدد نظام التقييم حالتان في هذا الأمر. Indian )

Green Building Council 2016)

الحالة الأولى : الفر اغات المعتمدة على التهوية الميكانيكية، وضع النظام جدول يوضـح الحد الادنى من معدل تغير الهواء

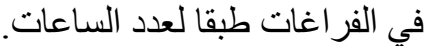

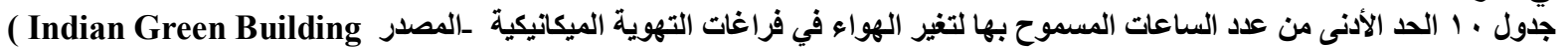
Council 2016)

\begin{tabular}{|c|c|}
\hline Type of space & Minimum out air change hour \\
\hline Recovery Room & 2 \\
\hline Critical \& Intensive care & 2 \\
\hline Trauma room & 3 \\
\hline Laboratory, sterilizing & 2 \\
\hline Medication room & 2 \\
\hline
\end{tabular}

الحالة الثانية الفراغات التي لا تعتمد على التهوية الميكانيكية بشكل أساسي، يجب توفير حد أدنى من مسطح الفتحات (الابو اب و الثبابيك) طبقا لمساحة الفر اغ للإمداد بالتهوية الطبيعية. 
جدول 11 الحد الادنى لمسطح القتحات طبقا لمساحة الفراغ ـالمصدر (Indian Green Building Council 2016 )

\begin{tabular}{|c|c|}
\hline Category & Percentage of Openable Area \\
\hline Regularly Occupied Area (<100 sq.m) & $8 \%$ \\
\hline Regularly Occupied Area (>100 sq.m) & $12 \%$ \\
\hline
\end{tabular}

ونفس الأمر بالنسبة للشرط الإلز امي الثاني و هو (التحكم في دخان التبخ الناتج عن التدخين)، إذا تم تحقيق هـاذان الشرطان

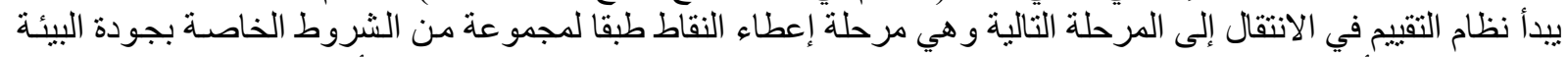

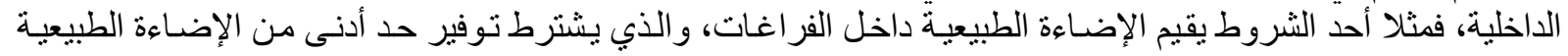

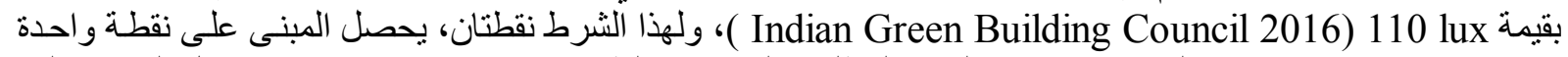

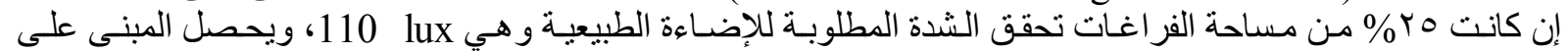

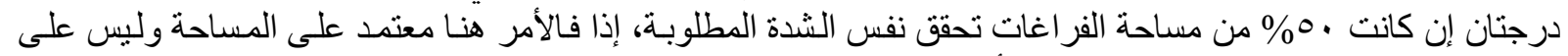

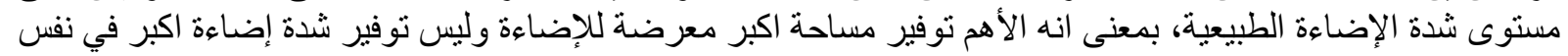

المساحة.

\section{جدول r I معدل توفير الإضاءة الطبيعة في الفراغات ـالمصدر (Indian Green Building Council 2016)}

\begin{tabular}{|c|c|c|}
\hline $\begin{array}{c}\text { Percentage of Patient area with } \\
\text { daylighting }\end{array}$ & $\begin{array}{c}\text { Percentage of other regularly } \\
\text { occupied spaces with daylighting }\end{array}$ & Points \\
\hline $25 \%$ & $25 \%$ & 1 \\
\hline $50 \%$ & $50 \%$ & 2 \\
\hline
\end{tabular}

حدد أيضا النظام شروط لتطبيق الشروط، أي انهه في الشرط السابق والمتعلق بتوفير الحد الأدنى من الإضـاءة الطبيعية،

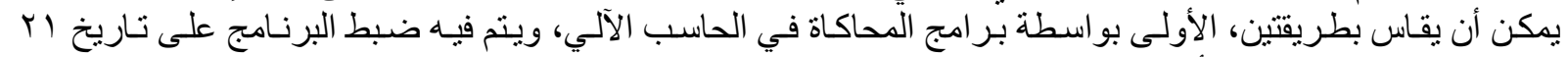

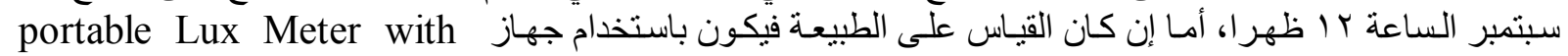
resolution of 10 lux

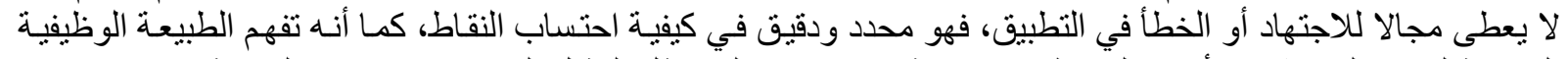

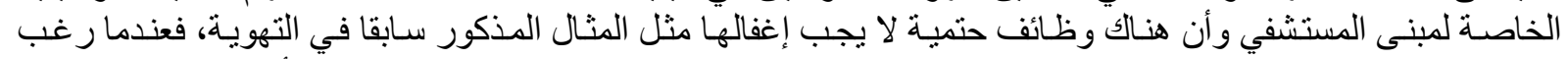

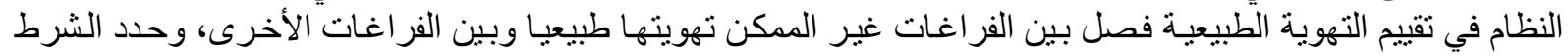

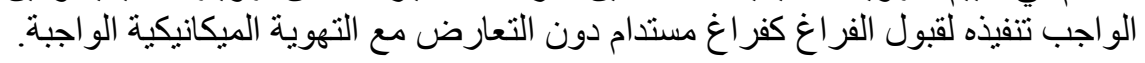

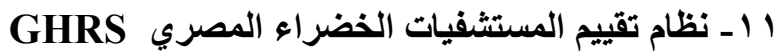

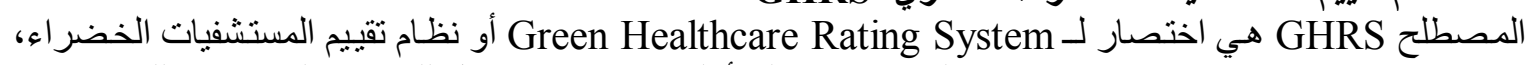

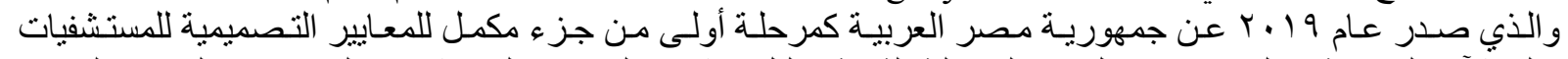

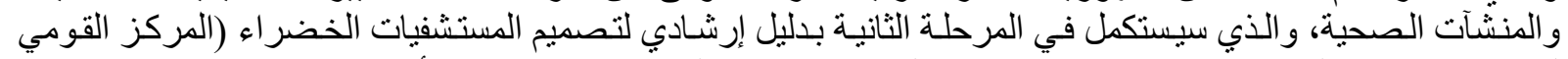

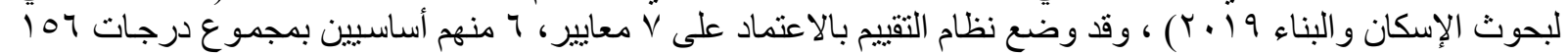

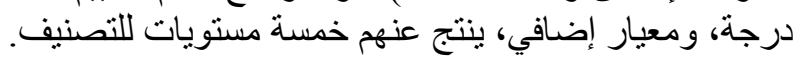

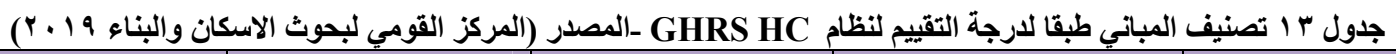

\begin{tabular}{|c|c|c|c|c|}
\hline بلاتيني & ذهبي & فضي & برونزي & معتمد \\
\hline \multicolumn{5}{|c|}{ يتم تحديد عدد النقاط والوزن النسبي لكل مستوى تبعا لاراء السادة الخبراء } \\
\hline
\end{tabular}




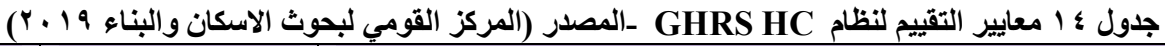

\begin{tabular}{|c|c|c|c|}
\hline النسبة المئوية & النقاط & المعيار & رقم \\
\hline$\% 11$ & IV & الموقع المستدام - إمكانية الوصول والعوامل البيئية & 1 \\
\hline$\%$ \%r & ro & كفاءة استخدام الطاقة & $r$ \\
\hline$\% 19$ & ro & كفاءة استخدام المياه & $r$ \\
\hline$\%$ IV & YV & المواد والموارد & $\varepsilon$ \\
\hline$\% 19$ & $r \cdot$ & جودة البيئة الداخلية وخلق بيئة استشفائية & $\bullet$ \\
\hline$\% 1 \leq$ & Yr & مجال الإدارة والتشغيل & 7 \\
\hline \multicolumn{2}{|c|}{ نقاط اضافية } & الابتكار & $\mathrm{V}$ \\
\hline$\% 1 \ldots$ & 107 نقطة & الاجمالى & \\
\hline
\end{tabular}

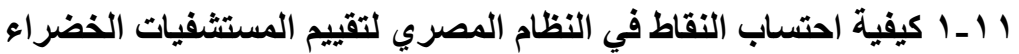

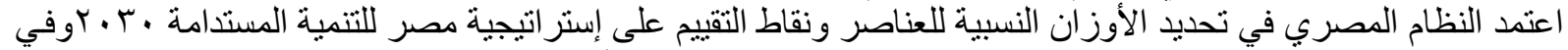

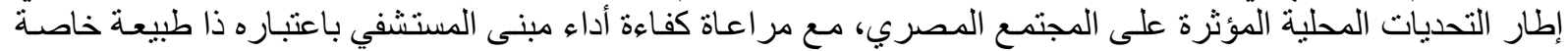

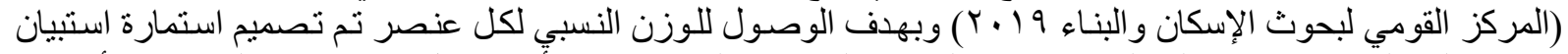

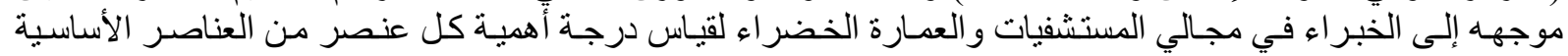

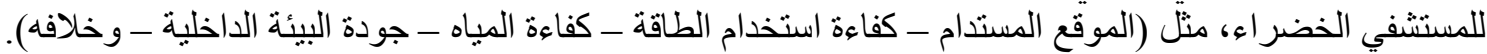

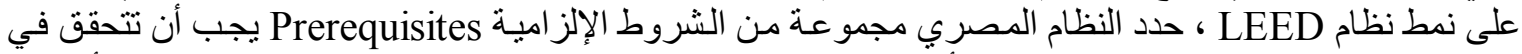

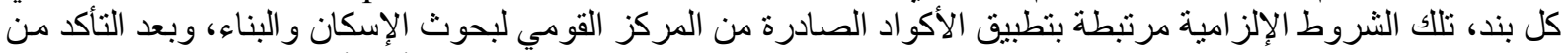

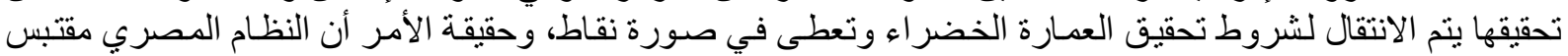

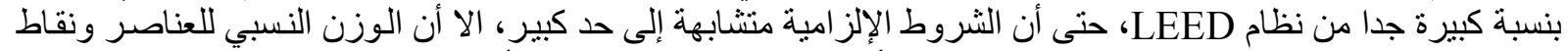
التقييم مختلفة في النظام المصري عن LEED، حيث أنها تحددت وفق استطلاع الر أي المشار إليه في الفقرة السابقة.

r ا إمقارنة بين نظم تقييم الاستدامة المستثفيات

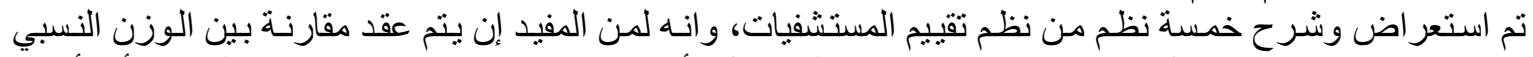

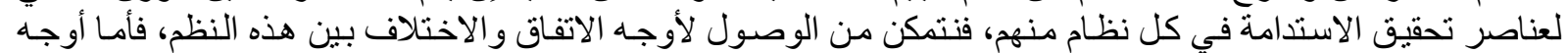

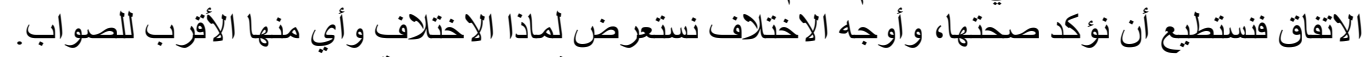

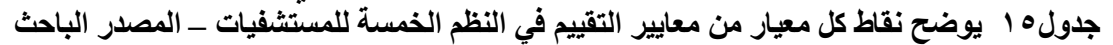

\begin{tabular}{|c|c|c|c|c|c|}
\hline $\begin{array}{l}\text { LEED } \\
\text { HC }\end{array}$ & $\begin{array}{l}\text { BREEAM } \\
\text { HC }\end{array}$ & $\begin{array}{l}\text { GREEN } \\
\text { STAR }\end{array}$ & $\begin{array}{l}\text { IGBC - } \\
\text { INDEA }\end{array}$ & $\begin{array}{l}\text { GHRS - } \\
\text { EGYPT }\end{array}$ & معيار التقييم \\
\hline 11 & 1. & $\Lambda$ & 11 & 11 & الموقع المستدام \\
\hline rq & 19 & $r \varepsilon$ & $r Y$ & $r Y$ & كفاءة استهلاك الطاقة \\
\hline 9 & 7 & Ir & 10 & 17 & كفاعة استهلاك المياه \\
\hline 19 & $1 Y .0$ & IV & 9 & IV & المواد والموارد \\
\hline \multirow[t]{6}{*}{11} & 10 & $r \cdot$ & Yr & 19 & جودة البيئة الداخلية ـ الصحة \\
\hline & Ir & 9 & & $1 \varepsilon$ & الادارة والتشغيل \\
\hline & $\Lambda$ & $\mathrm{V}$ & & & النقل \\
\hline & $1 \cdot$ & $\mu$ & & & التلوث والانبعاثات الضارة \\
\hline & V.0 & & & & النفايات \\
\hline & & & $1 \varepsilon$ & & التعقيم والنظافة \\
\hline 7 & 1. & Bonus & 0 & Bonus & الابتكار في التصميم \\
\hline$\varepsilon$ & & & & & الاولوية الاقليمية \\
\hline $1+1 \ldots$ & $1+1 \cdot \cdot$ & $1 \ldots$ & $1 \cdots$ & $1 \ldots$ & المجموع \\
\hline
\end{tabular}


من خلال المقارنـة السابقة يمكن استتناج عدة أمور ، على سبيل المثنال، يتضح أن الوزن الأكبر لكفاءة استهلاك الطاقة،

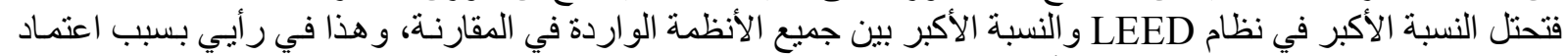

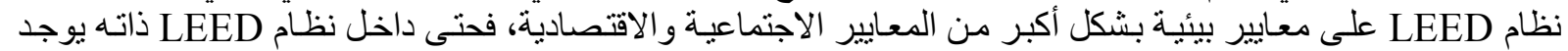

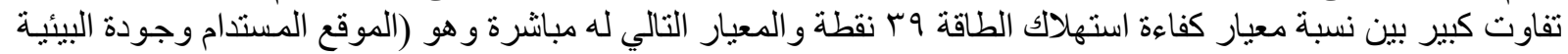

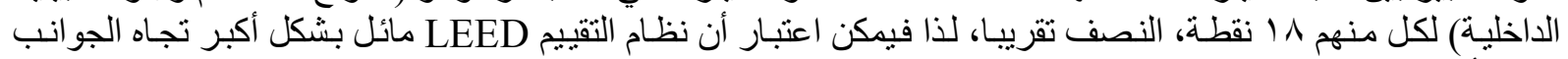
البيئية أكثر من الاجتماعية والاقتصنادية.

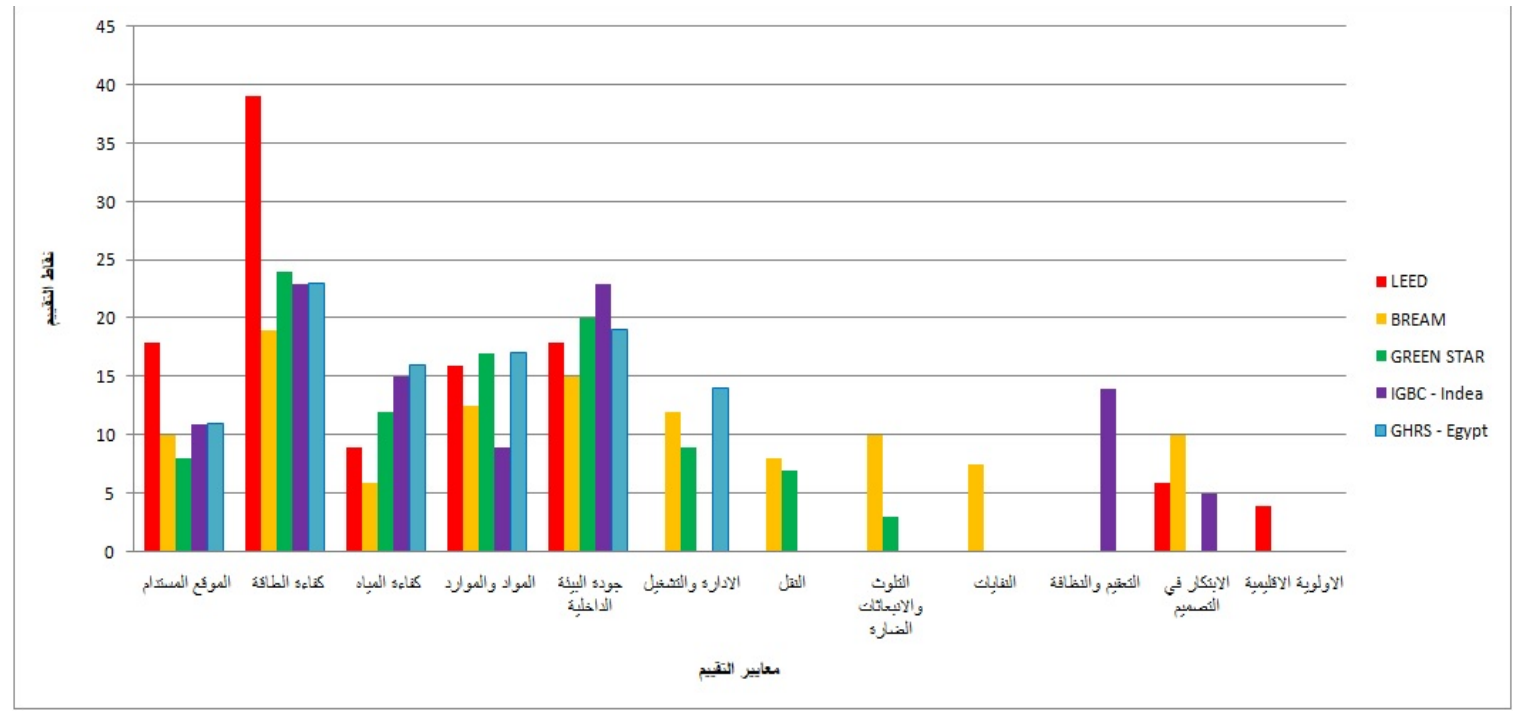

الشكل • رسم بياني للمقارنة بين نظم تقييم المستشفيات ـالمصدر الباحث

هناك بعض المعايير ظاهرة كمعايير منفصلة في بعض النظم وغير ظاهرة في بعض المعايير الأخرى، مثل معيار (النقل)

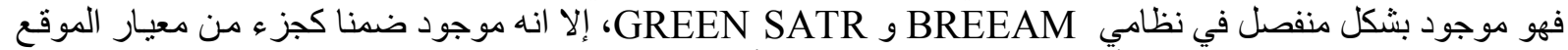

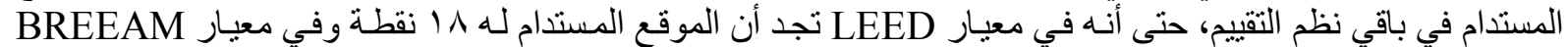

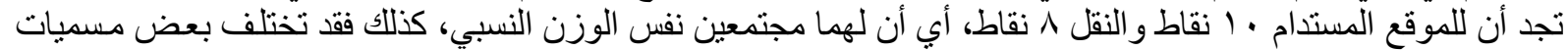

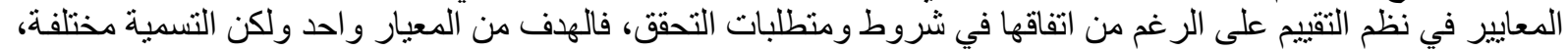

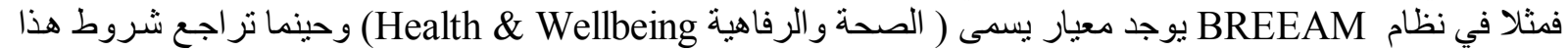

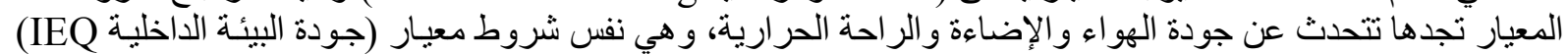
الموجودة في باقي نظم التقبيج.

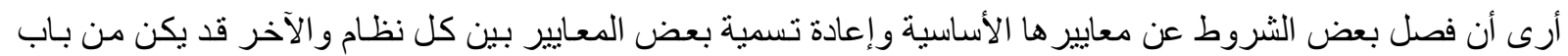

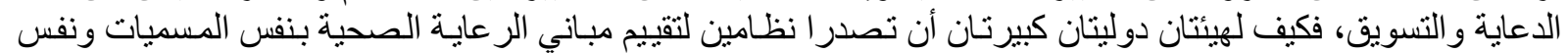

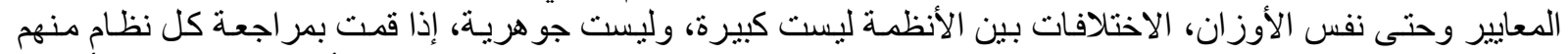

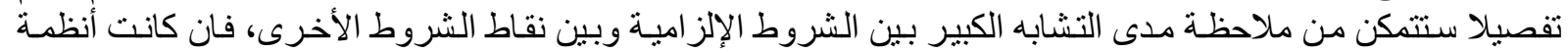

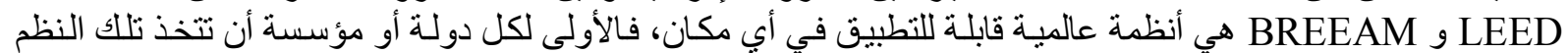

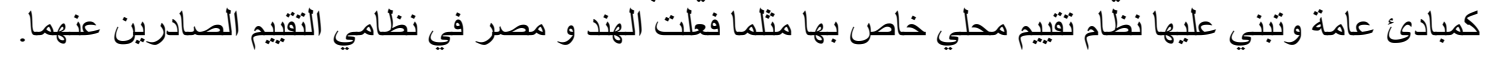

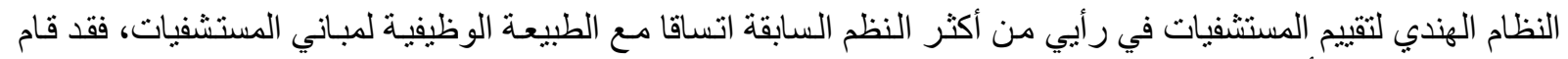

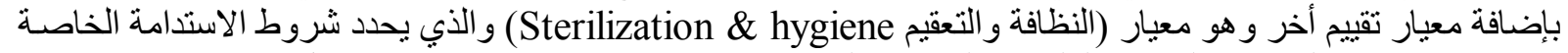

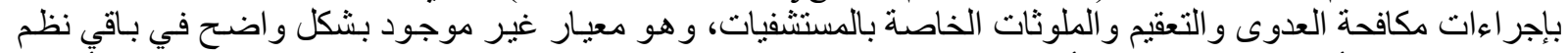

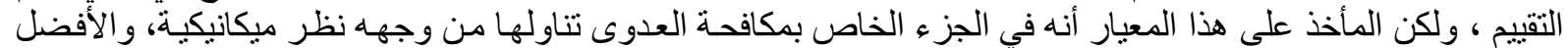

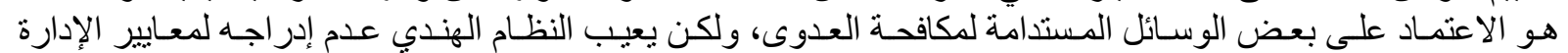
و التشغيل وكنلك التلوث و الانبعاث الضارة وبعض المعايير الأخرى التي أدرجت في باقي الأنظمة.

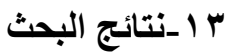

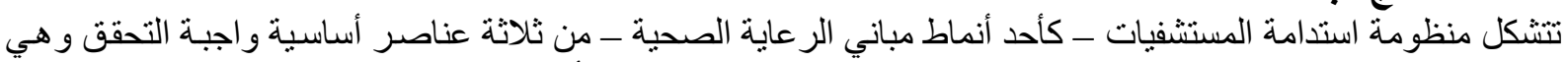

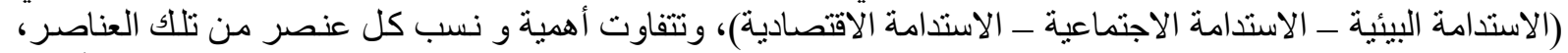

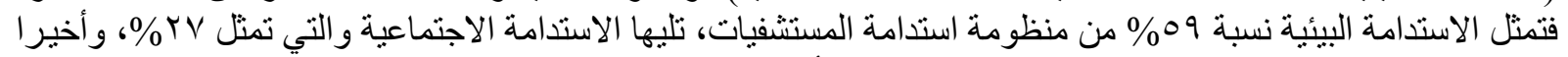
الاستدامة الاقتصادية و التي تمثل ع ( \% فقط و هي النسبة الأقل من الثلاثة عناصر. 


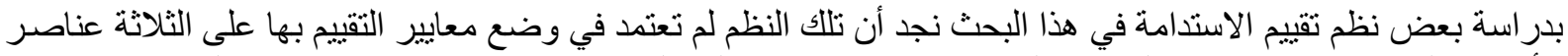

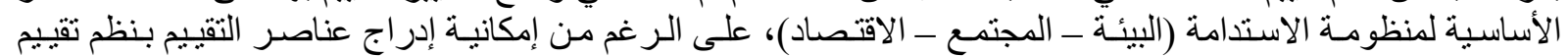

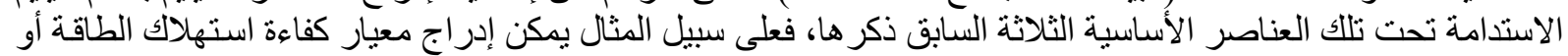

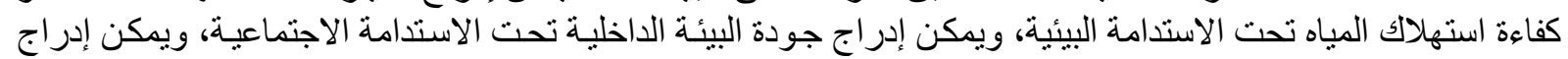
معيار الإدارة والتشغيل تحت الاستدامة الاقتصادية، ولكن لم تتبع نظم التقييم هذا النهج الموحد لونة لوضع المعايير الخاصة بها.

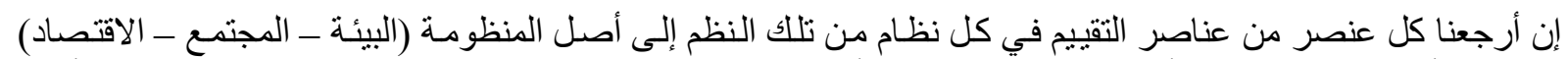

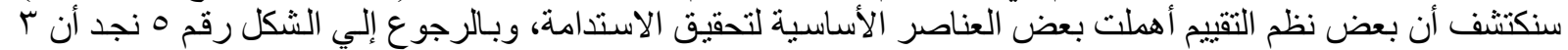
نظم فقط من ال 0 نظم هي من أدرجت معيار الإدارة و التشغيل الذي يمثل الاستدامة الاقتصادية الإنية ضمن معايير تقييمها،

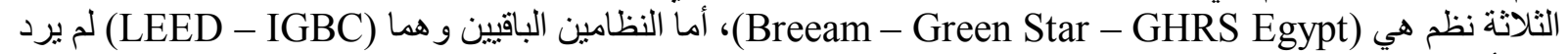
بهما أي شئ يخص الاستدامة الاقتصادية.

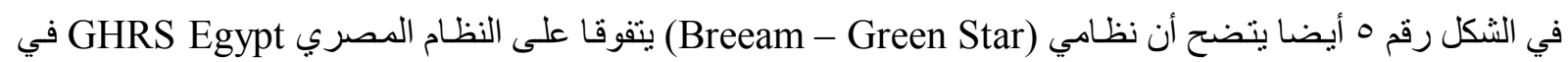

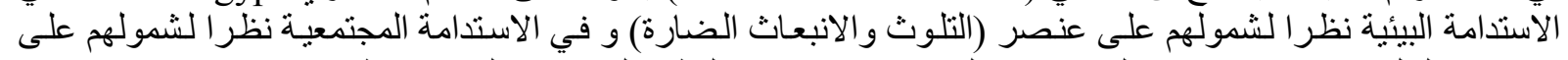

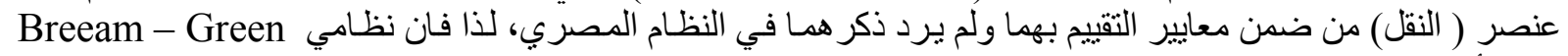
Star

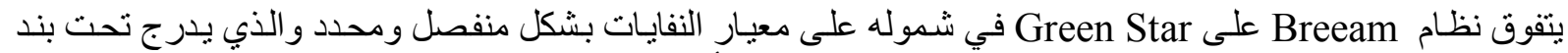

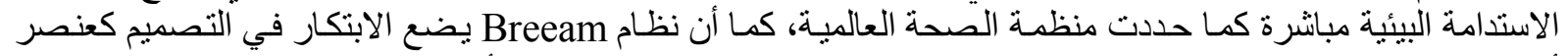

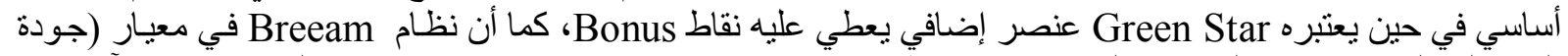

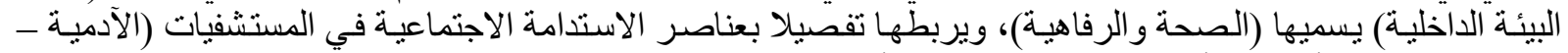

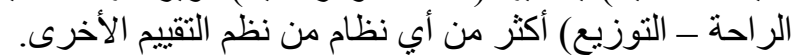

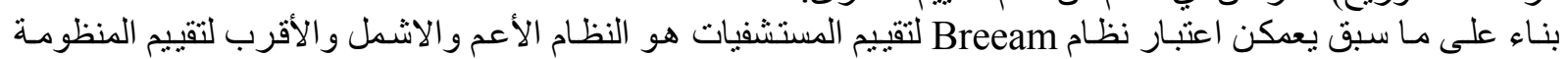
الكاملة لاستدامة المستشفيات بعناصن اعثر ها الثلاثة (البيئة ـ- المجتمع - الاقتصاد).

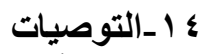

يوصى البحث بأن تتبع نظم التقييم العناصر الأساسية لاستدامة المستشفيات (البيئة ـ المجتمع ـ الاقتصاد)، وأن يكون نظسام

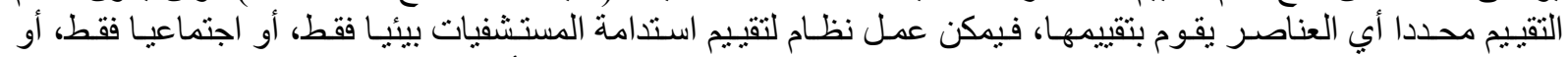

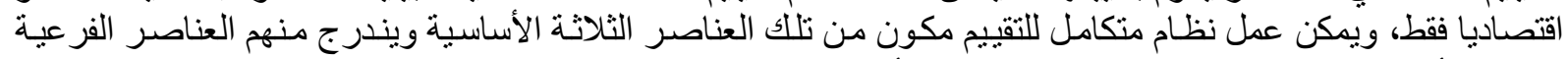

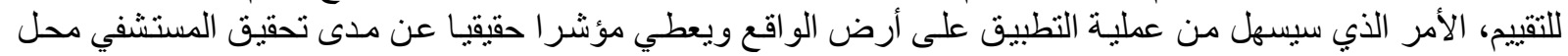
التقييم لعناصر منظومة الاستدامة كاملة.

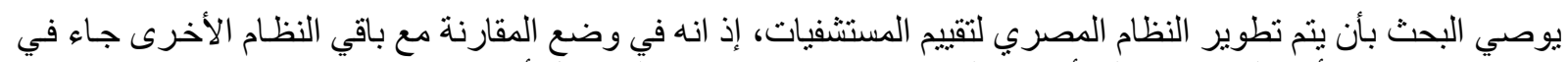

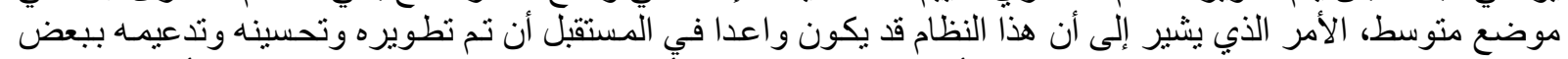

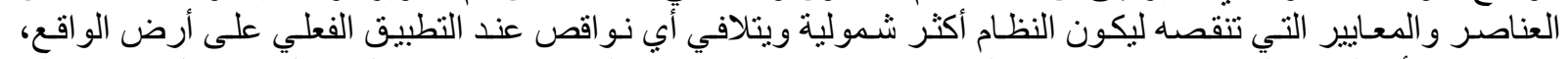

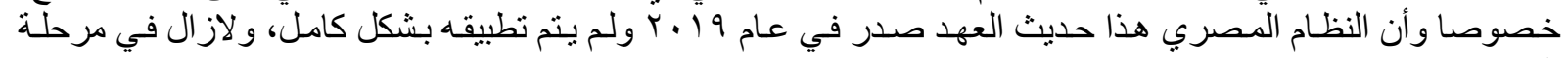
التجربة والاختبار.

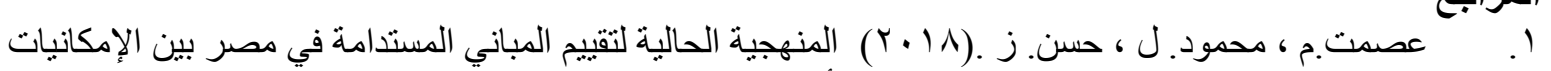

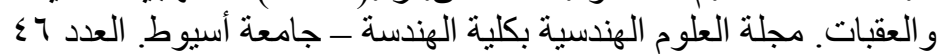

2. Bensalem, Sara. (2011) Sustainable Healthcare Architecture - Designing a Healing Environment. The University of Texas at Austin - School of Architecture. Center of Sustainable development

3. Daniels, S. R. (2006) the Consequences of Childhood Overweight and Obesity. The Future of Children.

4. Golbazi. M ,. Aktas. C. B (2016) Analysis of Credits Earned by LEED Healthcare Certified Facilities. International Conference on Sustainable Design, Engineering and Construction. ELSEVIER. Procedia Engineering 145 
5. Capolongo, Stefano \& others. Social sustainability in healthcare facilities: a rating tool for analyzing and improving social aspects in environments of care., Dipartimento di Architettura, IngegneriadelleCostruzioni e AmbienteCostruito (ABC), Politecnico di Milano, Via G. Ponzio 31, 20133 Milan, Italy. 2016 Vol.52

6. World Health Organization WHO.(2017) Environmentally sustainable health systems: a strategic document. The Regional Office for Europe of the World Health Organization . Copenhagen . Denmark

7. Heschong, L. .(2000) Thermal delight in architecture. Cambridge, Mass: MIT Press.

8. Liaropoulos ,L. Goranitis, I. (2015) Health Care Financing and Sustainability of Health Systems. International Journal of Equity in Health.

9. Björnberg A.(2013) Health consumer powerhouse, Euro Health Consumer Index Health Consumer Powerhouse.

10. U.S Green Building Council. (2012), LEED 2009 for HealthCare, Washington DC. USA

11. Buffoli. M \& Others. (2015) Healthcare Sustainability Evolution Systems. Springer International Publishing Switzerland.

12. BreGlobal. (2018) BREEAM UK - New Construction. Technical Manual. London. UK.

13. Australia Government..(2008) Australia 2020 Summit: Strengthening communities, supporting families and social inclusion, Canberra, Australia.

14. Xia. B \& Others. (2013) Green Star Points Obtained by Australian Building Projects. JOURNAL OF ARCHITECTURAL ENGINEERING C ASCE (American Society of Civil Engineers).

15. . Green Building Council. (2009) Green Star Healthcare V1.- Tool Fact Sheet \& Business Case. Australia.

16. Reed, R., Bilos, A., Wilkinson, S., and Schulte, K. W. (2009) International comparison of sustainable rating tools. J. Sustainable Real Estate.

17. Indian Green Building Council (2016) IGBC Green Healthcare Facilities Rating System.Pilot Versio . Abridged Reference Guide.

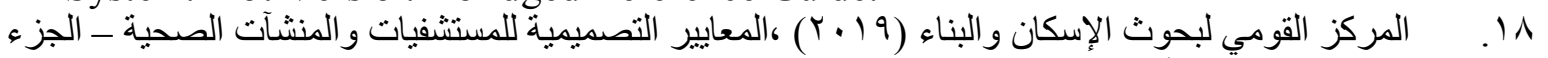

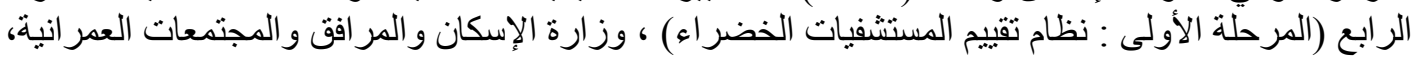

